ARTICLE

https://doi.org/10.1038/s41467-019-13075-8

\title{
Regulation of CHD2 expression by the Chaserr long noncoding RNA gene is essential for viability
}

Aviv Rom¹, Liliya Melamed', Noa Gil', Micah Jonathan Goldrich', Rotem Kadir², Matan Golan ${ }^{3}$, Inbal Biton ${ }^{4}$, Rotem Ben-Tov Perry ${ }^{1} \&$ Igor Ulitsky (i) ${ }^{1 \star}$

Chromodomain helicase DNA binding protein $2(\mathrm{Chd} 2)$ is a chromatin remodeller implicated in neurological disease. Here we show that Chaserr, a highly conserved long noncoding RNA transcribed from a region near the transcription start site of Chd2 and on the same strand, acts in concert with the CHD2 protein to maintain proper Chd2 expression levels. Loss of Chaserr in mice leads to early postnatal lethality in homozygous mice, and severe growth retardation in heterozygotes. Mechanistically, loss of Chaserr leads to substantially increased Chd 2 mRNA and protein levels, which in turn lead to transcriptional interference by inhibiting promoters found downstream of highly expressed genes. We further show that Chaserr production represses Chd2 expression solely in cis, and that the phenotypic consequences of Chaserr loss are rescued when Chd2 is perturbed as well. Targeting Chaserr is thus a potential strategy for increasing CHD2 levels in haploinsufficient individuals.

\footnotetext{
${ }^{1}$ Department of Biological Regulation, Weizmann Institute of Science, Rehovot, Israel. ${ }^{2}$ National Institute for Biotechnology in the Negev and Department of Microbiology, Immunology and Genetics, Ben-Gurion University of the Negev, Beer-Sheva, Israel. ${ }^{3}$ Department of Molecular Cell Biology, Weizmann Institute of Science, Rehovot, Israel. ${ }^{4}$ Department of Veterinary Resources, Weizmann Institute of Science, Rehovot, Israel. *email: igor.ulitsky@weizmann.ac.il
} 
T he mammalian transcriptome is highly complex, and contains tens of thousands of noncoding RNA genes ${ }^{1-4}$. A significant subset of these, referred to as long noncoding RNAs (lncRNAs), are at least 200 nucleotides (nt) in length, 3' polyadenylated, and $5^{\prime}$ capped, and are therefore structurally similar to mRNAs, but lack protein-coding potential ${ }^{5}$. Only a small portion of lncRNAs have been functionally characterized, and only very few of these have been studied in the context of organismal development ${ }^{6}$.

Chromodomain Helicase DNA Binding Protein 2 (Chd2) gene encodes an ATP-dependent chromatin-remodeling enzyme, which together with CHD1 belongs to subfamily I of the chromodomain helicase DNA-binding (CHD) protein family. Members of this subfamily are characterized by two chromodomains located in the $\mathrm{N}$-terminal region and a centrally located SNF2-like ATPase domain ${ }^{7}$, and facilitate disassembly, eviction, sliding, and spacing of nucleosomes ${ }^{8}$. There are conflicting reports on the genomic occupancy of CHD2. According to one report, based on ChIP-seq data obtained with antibodies against CHD1 and CHD2, both proteins bind predominantly in the proximity of gene promoters and share up to $60 \%$ of their DNA-binding sites in human cell lines ${ }^{9}$. Another study used MNase-ChIP-seq of endogenously tagged Chd1 and Chd2 in mouse embryonic stem cells (mESCs) ${ }^{10}$, and reported that the two proteins have different binding patterns-CHD1 binds predominantly to promoter regions, whereas $\mathrm{CHD} 2$ is associated with gene bodies of actively transcribed genes. CHD2 has also been linked to the deposition and incorporation of the $\mathrm{H} 3.3$ histone variant at transcriptionally active genes $9,11,12$ and at DNA damage sites, with the latter activity promoting repair of double-strand breaks ${ }^{13}$.

Mice homozygous for a gene-trap stop cassette in intron 27 of Chd2 survive until E18.5 with a marked growth retardation, and no viable offspring of these mice can be recovered ${ }^{14}$. Heterozygotes with this mutation show increased postnatal mortality at days $1-4$, and in the long term they exhibit growth retardation, shorter life spans, and altered morphology in various organs. However, a dominant negative effect of the truncated protein could not be excluded in this model. A different model for Chd 2 loss of function was recently created by the International Mouse Phenotyping Consortium, where exon 3 was replaced by a lacZ cassette and a stop signal ${ }^{15}$. No significant changes in mortality and aging were reported for these mice, but they exhibit slightly decreased body weight and length, skeletal abnormalities, abnormal bone structure, decreased fat amount and bone mineral density, and abnormalities in blood composition, such as decreased erythrocyte cell number, hemoglobin content, and mean platelet volume (http://www.mousephenotype.org/).

In humans, CHD2 haploinsufficiency is associated with neurodevelopmental delay, intellectual disability, epilepsy, and behavioral problems (reviewed in ref. ${ }^{16}$ ). Studies in mouse models and cell lines also implicate $C h d 2$ in neuronal dysfunction: perturbations of $C h d 2$ affect neurogenesis in the mouse developing the cerebral cortex ${ }^{17}$ and in human stem cells differentiated to neurons ${ }^{18}$, and loss of a single Chd 2 copy leads to deficits in neuron proliferation and a shift in neuronal excitability $^{19}$. Therefore, approaches for increasing CHD2 levels may have therapeutic relevance.

Multiple lines of evidence point to a strong link between lncRNA functions and those of chromatin-modifying complexes $^{20,21}$. Numerous chromatin modifiers have been reported to interact with $\operatorname{lncRNAs}{ }^{20}$. In addition, lncRNAs in vertebrate genomes are enriched in the vicinity of genes that encode for transcription-related factors ${ }^{2,22}$, including numerous chromatin-associated proteins, but the functions of the vast majority of these lncRNAs remain unknown. We hypothesized that the proximity of some lncRNA genes to genes involved in chromatin biology may imply a functional connection. To explore the biology of such interactions, we focus on one of the most conserved lncRNAs in vertebrates, found in close proximity to Chd2.

\section{Results}

Chaserr is a conserved IncRNA located upstream of Chd2. 1810026B05Rik in mouse (which we denote as Chaserr, for CHD2 adjacent, suppressive regulatory RNA) and LINC01578/ LOC100507217 in human (CHASERR), are almost completely uncharacterized lncRNAs, found upstream of and transcribed from the same strand as Chd2 (Fig. 1a). Chaserr has five exons, is polyadenylated, and is a bona fide lncRNA according to PhyloCSF 23 (Supplementary Fig. 1a), $\mathrm{CPAT}^{24}$ (coding probability 0.296), and $\mathrm{CPC}^{25}$ (coding potential score -1.23 ). According to FANTOM5 transcription start site (TSS) annotations, 3P-seq poly(A) site mapping, and RNA-seq data, Chaserr transcript is independent of Chd2 (Fig. 1a). The tandem organization with Chd2, Chaserr exon-intron structure, and parts of Chaserr sequence are conserved throughout vertebrates (Fig. 1a), which makes it one of the most conserved mammalian lncRNAs ${ }^{22,26}$. According to RefSeq annotation, the last exon of Chaserr in mouse overlaps Chd2; however, according to RNA-seq and 3P-seq data from various tissues, and to $3^{\prime}$ RACE in mouse embryonic fibroblasts (mEFs) (Supplementary Fig. 1b), the predominant Chaserr isoform ends $\sim 500$ bp after its last $3^{\prime}$ splice site, $\sim 2.2 \mathrm{~kb}$ upstream of the Chd2 TSS (as in GENCODE transcript ENSMUST00000184554), and we therefore considered this isoform in further studies. By using single-molecule RNA FISH, we found that the RNA product of Chaserr is mostly localized in the nucleus, in proximity to the $C h d 2$ site of transcription, and subcellular fractionation shows that it is enriched in the chromatin fraction (Fig. $1 \mathrm{~b}$ and Supplementary Fig. 1c). This nuclear enrichment is due at least in part to nonsense-mediated decay (NMD) that acts on Chaserr, likely triggered by a 117-codon non-conserved ORF that ends in the second exon (Supplementary Fig. 1d, e).

Chaserr and Chd 2 mRNA are tightly co-expressed across a panel of human and mouse tissues, and during mouse development according to ENCODE and FANTOM5 data (Fig. 1c and Supplementary Fig. 1f, g). Interestingly, both genes are expressed at appreciable levels in all studied samples, with particularly high expression in lymphocytes. The ratio between the expression levels of the two genes is also similar across the tissues, with notable exceptions of neuronal cells and fibroblasts, where Chaserr levels are relatively high (Fig. 1c and Supplementary Fig. 1f, g).

Chaserr is required for postnatal survival. In order to study its function, we generated Chaserr null alleles in mice by injection into fertilized oocytes of CRISPR/Cas 9 mRNA with a pair of gRNAs targeting sequences flanking the promoter and the first exon of Chaserr (Fig. 1d). This resulted in two different Chaserr $^{+/-}$mouse strains that carry deletions of 1032 and 1142 bps (Supplementary Fig. 1h), which were sufficient to abolish expression of mature Chaserr (see below), and the two lines were phenotypically indistinguishable from each other, and so we used them interchangeably in subsequent experiments. Strikingly, out of 38 pups born following crosses between Chaserr $^{+/-}$mice, we observed no Chaserr ${ }^{-/-}$pups. The numbers of Chaserr ${ }^{+/}$pups at weaning also deviated from expected Mendelian ratios ( $37 \%, P<10^{-8}$, Fig. 1e), and the surviving mice exhibited substantial growth retardation, occasional malocclusion, and neonatal lethality (Fig. 1f and Supplementary Fig. 1i-k). Pathological analysis of Chaserr ${ }^{+/}$mice revealed a wide range of abnormalities, including fat depletion, kyphosis, and thymic depletion, neither of which was highly penetrant. Out of 136 pups 
a

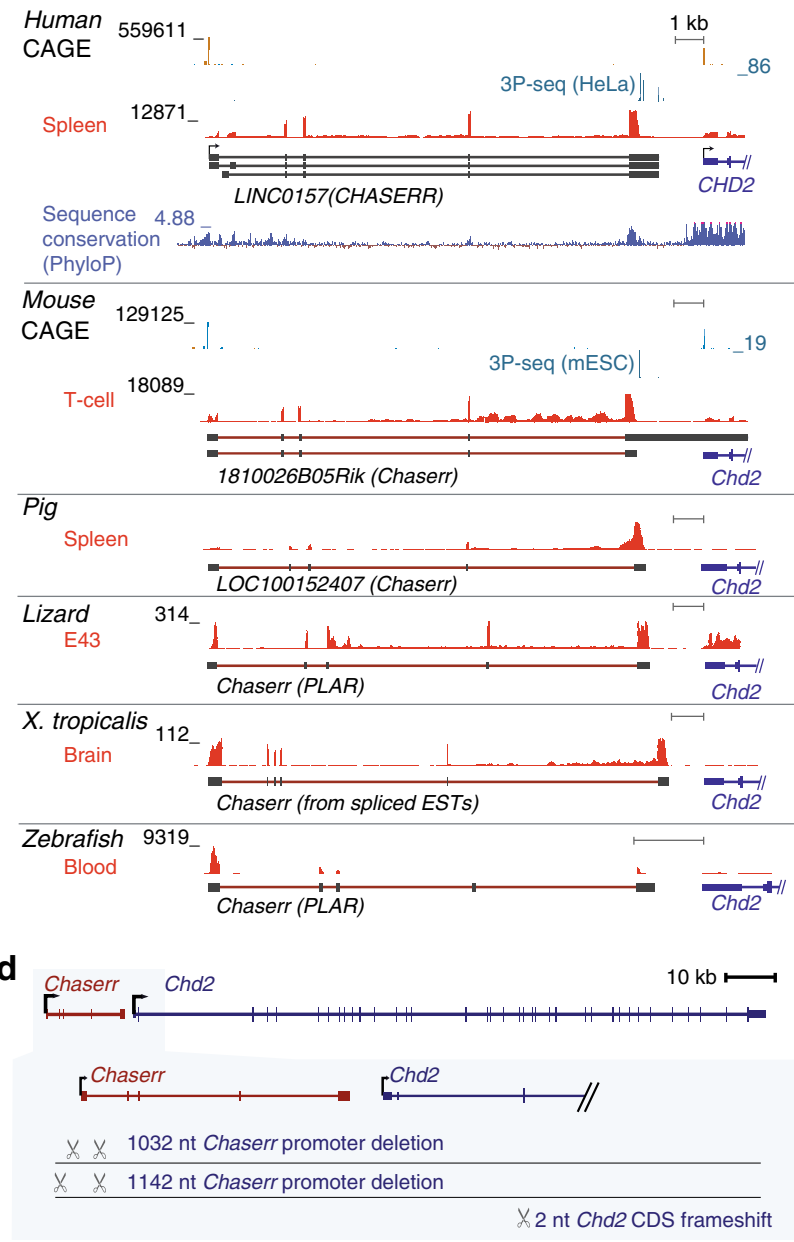

b
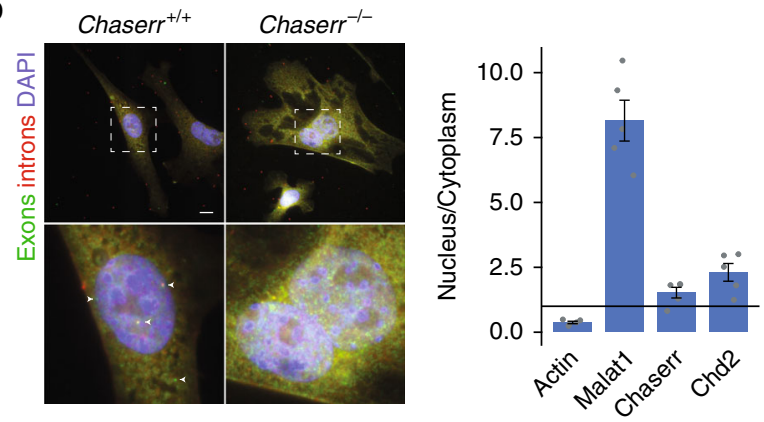

C
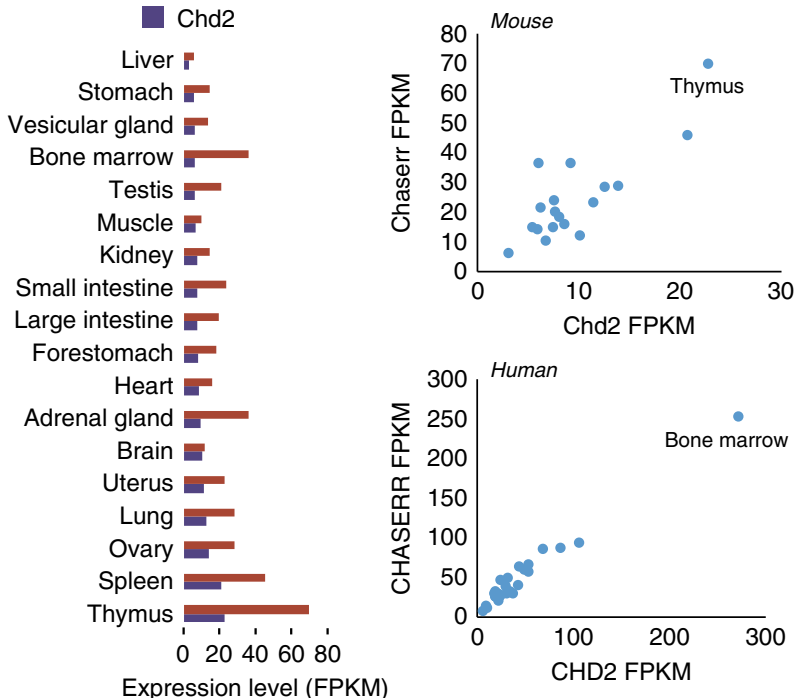

$\mathbf{f}$

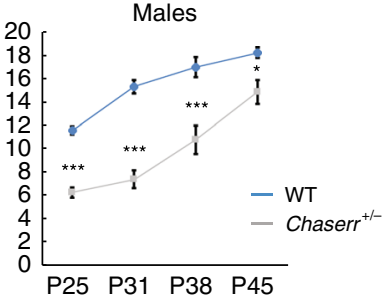

P25 $\quad$ P31 P38 P45 e

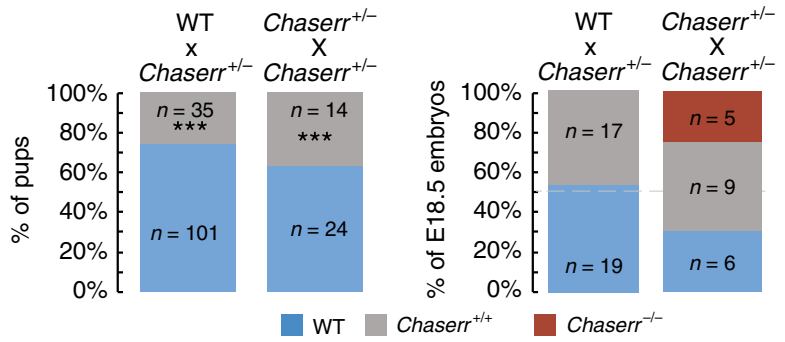

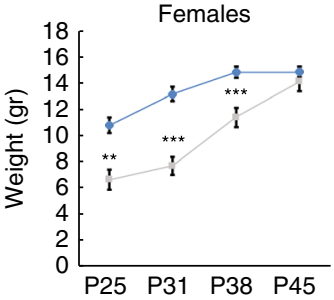

Fig. 1 Chaserr is essential for postnatal viability. a Genomic locus of Chaserr in the indicated vertebrate genomes. CAGE read coverage is from the FANTOM5 project ${ }^{75}$. 3P-seq data are from ref. ${ }^{76}$. RNA-seq data are from HPA ${ }^{77}$, ENCODE (mouse), FAANG (pig), SRP009831 (lizard), SRP039546 (xenopus), and SRP024369 (zebrafish). b Left: single-molecule FISH with probes targeting Chaserr introns (red) and exons (green) in mouse embryonic fibroblasts. Chaserr exons are marked by white arrowhead. Scale bar shows $10 \mu \mathrm{m}$. Right: qRT-PCR comparing cytoplasm and nuclear fractions in mESCs. $n=5$. c Left: Chaserr and Chd2 RNA expression across different tissues profiled in the mouse BodyMap ${ }^{78}$. Right: scatter plots for Chaserr and Chd2 expression in mouse BodyMap and human HPA ${ }^{77}$ projects. d Schematic of the positions of regions targeted by gRNAs used to generate Chaserr ${ }^{-/-}$and $C h d 2 \mathrm{~m} / \mathrm{m}$ mice. e Left: neonate survival rates for the indicated crossing. Right: embryonic survival rates at the indicated embryonic time points. $\mathbf{f}$ Weights of neonates at the indicated age (males $n=7-15$, females $n=6-18$ per group). Error bars show S.E.M. ${ }^{\star} P<0.05,{ }^{\star \star} P<0.01$, and ${ }^{\star \star \star} P<0.001$ (e chi-squared test. $\mathbf{f}$ two-sided $t$ test). Source data are provided as a Source Data file

born from Chaserr ${ }^{+/}$and Chaserr ${ }^{+/+}$crosses, 35 were Chaserr $^{+/}(\sim 25 \%)$, significantly less than the expected $50 \%(P<$ $10^{-5}$, Fig. 1e), suggesting that two copies of Chaserr are required for proper postnatal survival. In contrast, embryos were recovered with the expected Mendelian ratios in crosses between Chaserr ${ }^{+/-}$ and Chaserr ${ }^{+/+}$mice and in Chaserr ${ }^{+/}$intercrosses (Fig. 1e and Supplementary Fig. 11), suggesting that Chaserr is required for postnatal, but not embryonic, survival. Chaserr ${ }^{+/}$females very rarely became pregnant, and therefore we focused on crosses between Chaserr ${ }^{+/+}$females and Chaserr ${ }^{+/}$males for most of this study.

Due to the close proximity and co-expression of Chaserr and Chd2, we tested whether Chd2 expression is affected in Chaserr $^{+/}$embryos. Chd 2 mRNA was significantly upregulated by $\sim 1.5$-fold during embryonic development at the examined time points (E9.5, E10.5, and E13.5), in mEFs derived from E13.5 
a

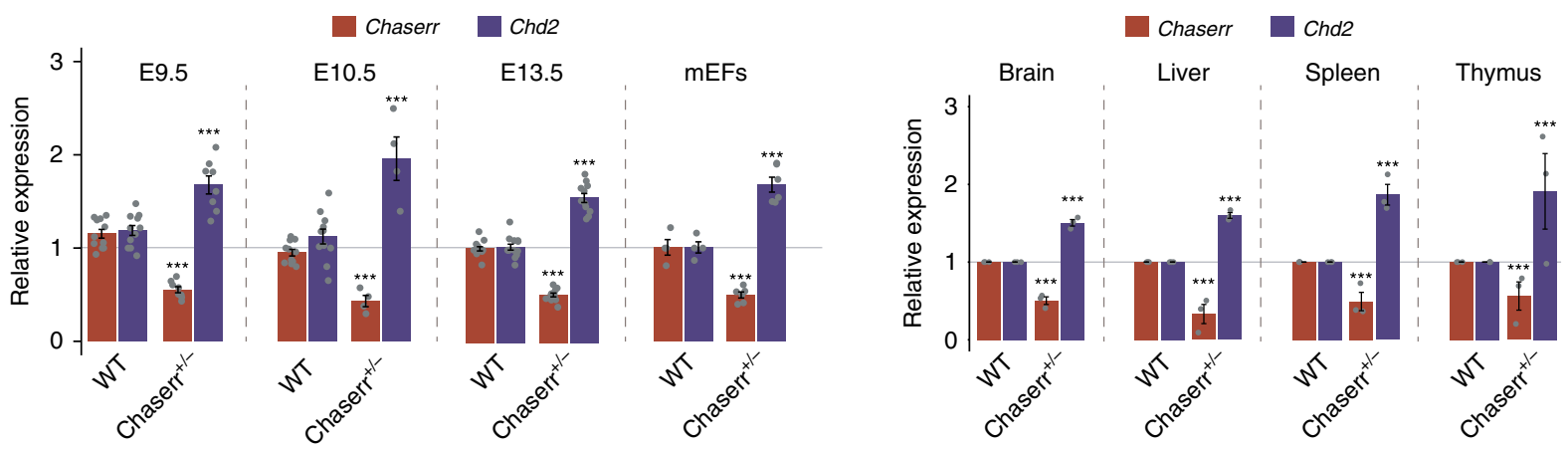

b
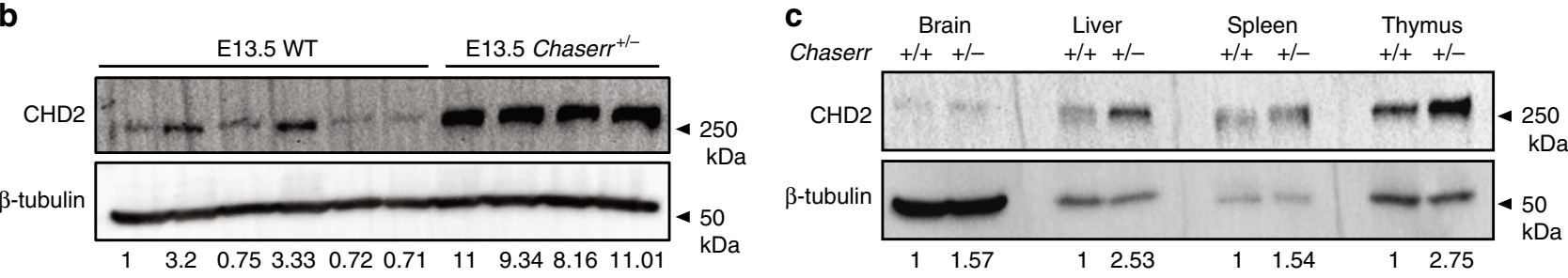

d
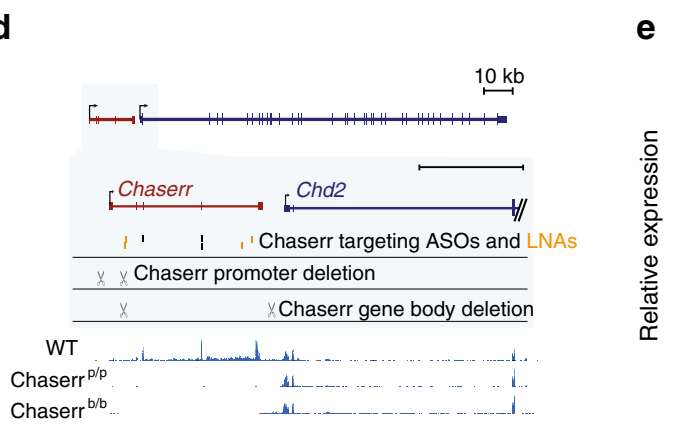

e

Chase

Chaserr Chd2

f

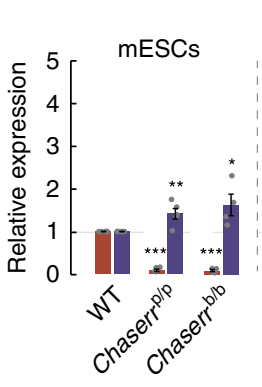

mESC-derived

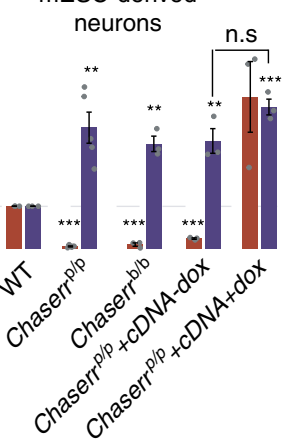

Neuro2a
cells

Chaserr $^{\mathrm{b} b}$

- 1
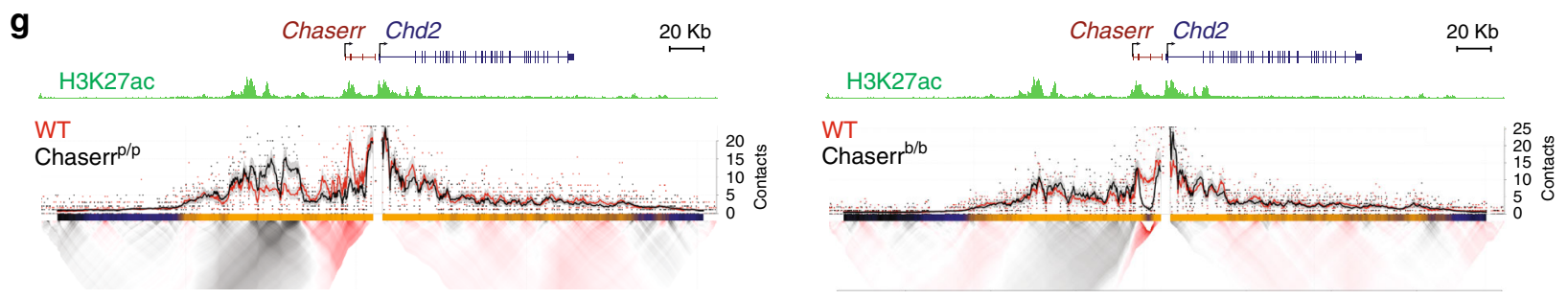

Fig. 2 Chaserr represses Chd2 expression. a qPCR of the indicated genes in whole embryos from the indicated developmental stage, mEFs, or adult tissues with the indicated background. $n$ for each group of embryos or mEFs is indicated in parentheses. Normalized to Actb. $n=3$ for the tissues. $\mathbf{b}$ Western blot for the indicated protein in individual E13.5 whole embryos from indicated background. $\mathbf{c}$ As in $\mathbf{b}$ for the adult tissues. $\mathbf{d}$ Top: scheme of regions targeted by gRNAs used to generate Chaserrp/p, Chaserr $\mathrm{b} / \mathrm{b} \mathrm{mESCs}$, or the antisense reagents; Bottom: RNA-seq read coverage in mESCs from the indicated background. e, $\mathbf{f}$ qRT-PCR of the indicated genes in the indicated backgrounds or treatments. Normalized to Actb. $n=3-5$. $\mathbf{g}$ Targeted Chromosome Conformation Capture (4C) analysis with the Chd2 promoter as the viewpoint in WT vs. Chaserrp/p (left) or WT vs. Chaserrb/b (right) mESCs. Top to bottom: scheme of the Chd2-Chaserr locus; H3K27ac ChIP-seq signal in mESCs; smoothed trend lines and raw counts of the contact profiles in the indicated cell lines; domainogram ${ }^{79}$ showing mean contact per fragment end for a series of window sizes. Ratio quantifications are shown below Western blots. Source data are provided as a Source Data file. Error bars show S.E.M. ${ }^{\star} P<0.05,{ }^{\star \star} P<0.01$, and ${ }^{\star \star \star} P<0.001$ (a, e, f: two-sided $t$ test)

embryos, and in four adult tissues (Fig. 2a). Western blot (WB) in E13.5 embryos and in the adult tissues also demonstrated strong upregulation of CHD2 protein (Fig. 2b, c).

Chaserr RNA or its transcription repress Chd2. As Chaserr-/mice could not be generated, in order to study the regulation of Chd2 in Chaserr ${ }^{-1-}$ cells and efficiently compare different Chaserr perturbations, we used CRISPR/Cas9 in mouse embryonic stem cells (mESCs) to engineer clones with either homozygous loss of
Chaserr promoter and the first exon (Chaserrp/p), or deletion of the rest of the Chaserr gene body from the first intron to just downstream of Chaserr polyadenylation site (Chaserr b/b) (Fig. 2d). Importantly, the Chaserr ${ }^{\mathrm{b} / \mathrm{b}}$ line has an intact Chaserr promoter, which still recruits RNA Pol2 (Supplementary Fig. 2a). Chaserr expression was abolished in both mutant lines, as evident in RNA-seq data (Fig. 2d), leading to significant Chd2 mRNA upregulation (Fig. 2e). This upregulation is consistent with a previous study that examined Chaserr as part of a panel of 
lncRNAs (Chaserr was referred to as linc2025 in that study) and also observed an increase in Chd2 upon deletion of Chaserr promoter $^{27}$. Chd2 was also significantly upregulated in neurons derived from Chaserr $\mathrm{p} / \mathrm{p}$ and Chaserr $\mathrm{b} / \mathrm{b}$ mESCs (Fig. 2e). Similarly, targeting of Chaserr in Neuro2a cells by using antisense oligonucleotides (ASOs) or LNA Gapmers reduced Chaserr expression by $\sim 75 \%$, and led to a significant increase in Chd 2 mRNA and protein levels (Fig. 2e and Supplementary Fig. 2b, c). Furthermore, induction of Chaserr expression from the endogenous locus by using CRISPR activation ${ }^{28}$ resulted in a decrease in Chd2 mRNA levels (Fig. 2f). We note that other perturbation methods, like insertion of polyA sites ${ }^{29}$ are not effective for perturbing Chaserr ${ }^{27}$. We conclude that an intact Chaserr represses Chd2 expression in all the systems we studied.

Importantly, Chaserr $\mathrm{b} / \mathrm{b}$ mESCs have an intact Chaserr promoter, which recruits RNA Pol2 (Supplementary Fig. 2a), and thus this line helps address the possibility of a competition between the Chaserr and Chd2 promoters over shared enhancers. Indeed, Targeted Chromosome Conformation Capture (4C) analysis of the two lines showed that while deletion of Chaserr promoter resulted in a pronounced increase of contacts between the Chd2 promoter and an array of enhancers upstream of the Chaserr/Chd2 locus, no substantial changes were observed in the Chaserrb/b cells (Fig. 2g), suggesting that enhancer competition is not the predominant cause of the increased Chd2 levels in these cell lines.

In order to test whether the mature Chaserr RNA is sufficient for repression of Chd2, we infected the Chaserrp/p mESCs with a lentivirus carrying a doxycycline (dox)-inducible Chaserr cDNA. Chaserr was overexpressed relative to the WT levels by $\sim 3.5$-fold following Dox addition, but Chd2 mRNA expression was not affected compared with no-Dox control (Fig. 2e). We conclude that either the RNA product of Chaserr, or transcription from its endogenous locus (which might be affected by the ASO/Gapmer cleavage of the nascent transcript), are required for repression of Chd2 expression.

Chaserr acts in cis. We next examined whether the early lethality of mice with Chaserr loss of function is mediated by increased Chd 2 levels. We used CRISPR/Cas9 with a single gRNA targeted to the third exon of Chd2 (Fig. 1d and Supplementary Fig. 2d) to generate $C h d 2^{\mathrm{m} / \mathrm{m}}$ mice that had a 2-bp deletion in the coding sequence of Chd2. This mutation had a mild effect on Chd2 mRNA expression, but dramatically reduced CHD2 protein levels, as expected from a frameshift in the main coding frame, with the residual protein possibly emanating from an alternative start codon (Fig. 3a, note that the monoclonal antibody we used recognizes a peptide in the C-terminal part of CHD2). Chd $2^{\mathrm{m} / \mathrm{m}}$ mice were born at expected Mendelian ratios (Supplementary Fig. 2e), and similarly to the $C h d 2^{\mathrm{tm}} \mathrm{lb}$ (EUCOMM)Hmgu mice generated by insertion of a LacZ cassette into the second intron and deletion of the third exon of $C h d 2^{30}$, had mild reduction in size and in subcutaneous adipose tissue, but no gross developmental or fertility phenotypes. This enabled us to breed Chaserr ${ }^{+/-}$mice with $C h d 2^{\mathrm{m} / \mathrm{m}}$ mice (Fig. 3b). We intercrossed the resulting Chaserr ${ }^{+/-} C h d 2^{\mathrm{m} /+}$ offspring, and out of 44 pups born from such crosses, only 14 ( 32\%) were Chaserr+/- (Supplementary Fig. 2f), suggesting that one hypomorphic allele of Chd2 is not sufficient for compensating for loss of one Chaserr allele. Indeed, CHD2 protein levels were substantially higher in Chaserr ${ }^{+/-} C h d 2^{\mathrm{m} /+}$ mice when compared with their Chaserr ${ }^{+/+} \mathrm{Chd} 2^{\mathrm{m} /+}$ littermates (Fig. 3c), potentially explaining the neonatal lethality and growth retardation (Supplementary Fig. 2g).

The Chaserr ${ }^{+l-} C h d 2^{\mathrm{m} /+}$ offspring that survived allowed us to test with allele-specific qRT-PCR whether Chaserr affected Chd2 expression from the cis allele in vivo (Supplementary Fig. 2h).
Only Chd2 in cis to the Chaserr ${ }^{-}$allele was upregulated in Chaserr ${ }^{+/}$Chd $2^{\mathrm{m} /+}$ mice when compared with Chaserr ${ }^{+/+}$ Chd $2^{\mathrm{m} /+}$ littermates (Fig. 3d), suggesting that Chaserr represses Chd2 through cis-acting regulation. Interestingly, the Chd2 mRNA produced from the Chaserr ${ }^{+}$allele was repressed compared with WT levels, hinting at a possible feedback regulation resulting from excess CHD2 expression (see Discussion). mEFs derived from E13.5 Chaserr ${ }^{+/}$Chd $2^{\mathrm{m} /+}$ embryos allowed us to test whether the effect of knocking down Chaserr on Chd2 also occurs strictly in cis. Indeed, treatment of these mEFs with LNA Gapmers targeting Chaserr (which is expressed only from the $C h d^{\mathrm{m}}$ allele) resulted in an increase in expression of Chd ${ }^{m}$ mRNA, but did not affect $C h d^{+}$mRNA levels (Fig. 3e), further solidifying the cis-acting function of Chaserr RNA product or the act of its transcription.

Chd2 loss of function rescues phenotypes of Chaserr-/- mice. In order to directly test whether the severe Chaserr loss-of-function phenotype is mediated by CHD2 overexpression, we used CRISPR/Cas9 to delete the Chaserr promoter and the first exon in a $C h d 2^{\mathrm{m} / \mathrm{m}}$ mouse (Supplementary Fig. 3a), and thus generated a model in which loss of Chaserr increases the expression of a hypomorphic allele of Chd2. Out of 137 pups born from intercrosses of Chaserr ${ }^{+/}$Chd $2^{+/ \mathrm{m}}$ mice, 38 were Chaserr ${ }^{-/}$ Chd2 $2^{\mathrm{m} / \mathrm{m}}(\sim 27 \%), 58 \mathrm{Chaserr}^{+/-} \mathrm{Chd} 2^{+/ \mathrm{m}}(\sim 42 \%)$, and $41 \mathrm{WT}$ $(\sim 30 \%)$, which did not deviate significantly from normal Mendelian ratios $(\mathrm{P}=0.2$; Fig. $3 \mathrm{f}$ and Supplementary Fig. $3 \mathrm{~b}, \mathrm{c})$, despite up to sixfold upregulation of Chd2 mRNA in different tissues of Chaserr ${ }^{-1}$ Chd2 $2^{\mathrm{m} / \mathrm{m}}$ mice (Fig. 3g). Despite Chd2 mRNA upregulation, CHD2 mutant protein levels in tissues from Chaserr ${ }^{-1} \mathrm{Chd}^{\mathrm{m}} / \mathrm{m}$ mice were not higher than the WT protein levels in WT mice (Fig. 3h). Chaserr ${ }^{-1-}$ Chd $2^{\mathrm{m} / \mathrm{m}}$ and Chaserr ${ }^{+1-}$ Chd $2^{+/ \mathrm{m}}$ mice showed no significant differences in weight (Fig. 3i and Supplementary Fig. 3d), and no obvious phenotypes, in stark contrast to the common and pleiotropic phenotypes observed in Chaserr $^{+/-}$neonates on $\mathrm{Chd2^{+/+ }}$ background. A hypomorphic allele of $C h d 2$ can thus rescue the lethality caused by loss of Chaserr, when the two occur on the same allele.

To further characterize the changes in levels of Chd2 mRNA in the double mutants, we isolated mEFs from E13.5 embryos from Chaserr $^{+/+}$, Chaserr ${ }^{+/}$, and Chaserr ${ }^{-1-}$ genotypes on Chd $2^{\mathrm{m} / \mathrm{m}}$ background. qRT-PCR analysis showed that Chaserr expression is completely abolished in Chaserr ${ }^{-1-} C h d 2^{\mathrm{m} / \mathrm{m}}$ mice, and that Chd2 mRNA was significantly overexpressed in a Chaserr dosagedependent manner (Fig. 3j).

Chaserr loss leads to extensive transcriptional changes. Having established that the severe phenotype resulting from Chaserr loss is mediated by CHD2, we were next interested to understand the consequences on gene expression of Chaserr loss and CHD2 upregulation. Beyond the increase in Chd2 mRNA levels, limited changes in gene expression were observed in Chaserr ${ }^{+/-}$E9.5 or E13.5 embryos, mEFs, or brains (Supplementary Fig. 4a). In contrast, hundreds of genes were differentially expressed in Chaserr ${ }^{-1} \mathrm{mEFs}$, which showed a more than two-fold increase in Chd2 mRNA and protein levels (Fig. 4a). Changes in gene expression in three independently-derived Chaserr ${ }^{-1-} \mathrm{mEFs}$ were strongly correlated with those observed in an E13.5 Chaserr ${ }^{-/}$ embryo $\left(R=0.4, P<10^{-15}\right)$. The 1493 significantly downregulated genes (down by at least $25 \%$, adjusted $P<0.05$ ) were enriched for various GO categories, such as nervous and connective tissue development and transcriptional regulation, and were very significantly enriched for genes whose loss of function in mice is associated with phenotypes such as decreased length of long bones, decreased body weight, respiratory distress, and 
a

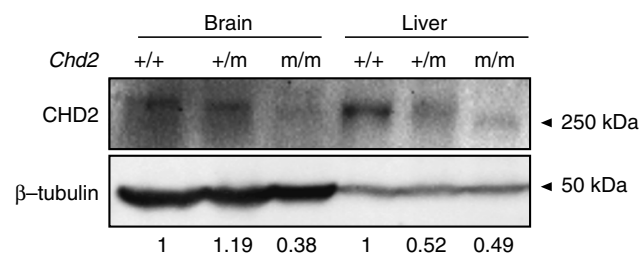

c

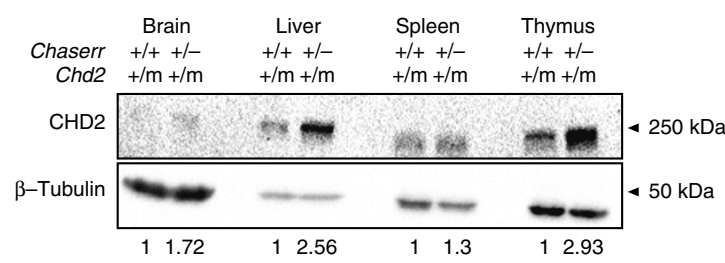

b

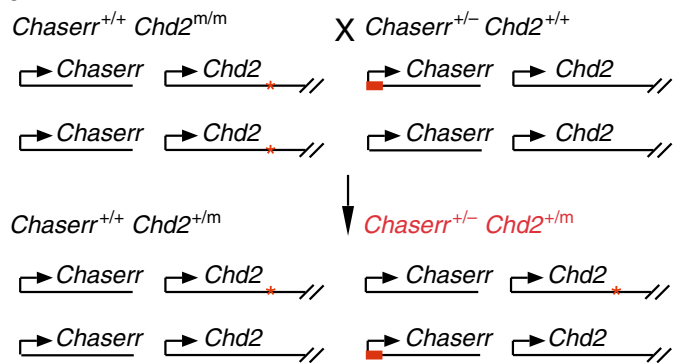

f

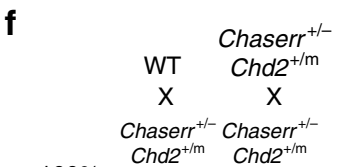

i

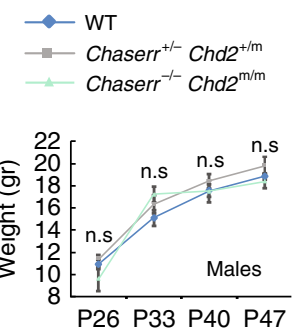

$\mathbf{j} \backsim \mathrm{WT}$

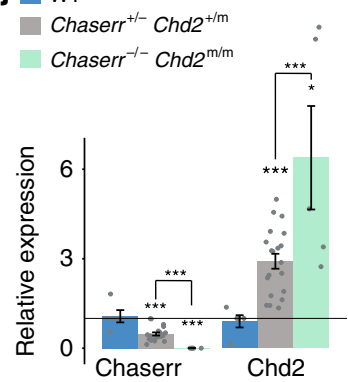

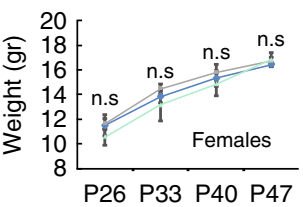

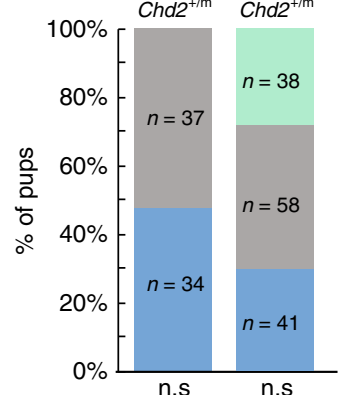

P26 P33 P40 P47 g

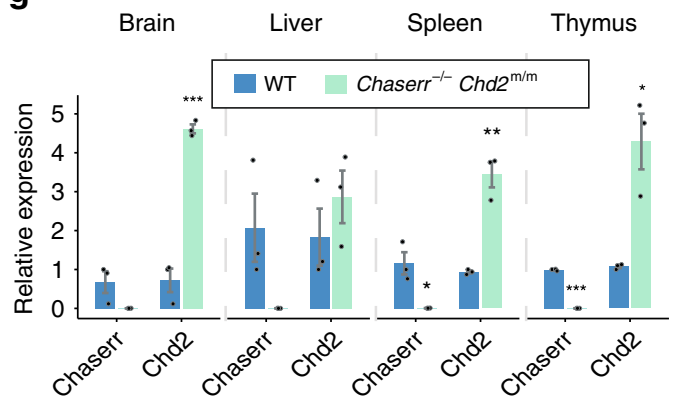

h

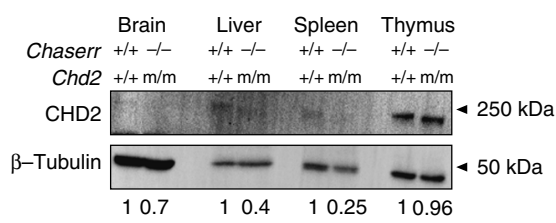

e

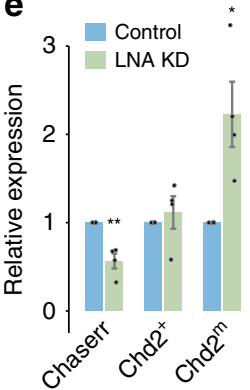

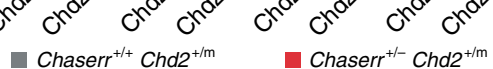

Fig. 3 Chaserr regulates Chd2 expression in cis. a CHD2 Western blot in the indicated tissues from mice with the indicated genotypes. $\mathbf{b}$ Scheme of the cross between Chaserr ${ }^{+/-}$and Chd $2^{\mathrm{m} / \mathrm{m}}$ mice. c Western blot in the indicated tissues from mice with the indicated genotypes. $\mathbf{d}$ Chd2 allele-specific expression in the indicated backgrounds in four different mouse tissues. Normalized to Actb. $n=3$ for the indicated tissues. e Changes in expression of Chaserr, or of Chd2 mRNA from the indicated allele, in Chaserr $+/-$ Chd2 $\mathrm{m} /+\mathrm{mEFs}$ transfected with a mix of two LNAs targeting Chaserr or non-targeting control (NT). $\mathbf{f}$ Neonate survival rates for the indicated crossing. $\mathbf{g}$ qRT-PCR in the indicated adult tissues and backgrounds. Normalized to Actb. $n=3$ for the indicated tissues. h CHD2 Western blot from the indicated tissues and backgrounds. i Pup weights for the Chaserr $+/-C h d 2 \mathrm{~m} /+$ intercross. $n=5-19$ pups per genotype per time point. j qRT-PCR for mEFs with the indicated background. Normalized to Actb. WT $n=5, \mathrm{Chaserr}+/-\mathrm{Chd}_{2}+/ \mathrm{m} n=20$, Chaserr ${ }^{+/}-$Chd $2^{+/ m} n=5$. Error bars show S.E.M. ${ }^{\star} P<0.05,{ }^{\star \star} P<0.01$, and ${ }^{\star \star \star} P<0.001$ (d, e, g, i, j: two-sided $t$ test. f: chi-squared test). Ratio quantifications are shown below Western blots. Source data are provided as a Source Data file

premature death (Supplementary Data 1), closely related to the phenotypes observed in our Chaserr loss-of-function model. Specifically, some of the most downregulated genes, such as Ctsk, Cpt1c, and Dapk3, which were also validated by qRT-PCR (Fig. 4c), are known to be required for proper embryonic development $^{31}$. In contrast, the 616 significantly upregulated genes (up by at least $25 \%$, adjusted $P<0.05)$ were enriched for various RNA-related GO categories (Supplementary Data 1).

Gene expression changes in Chaserr ${ }^{-1}$ mEFs were significantly inversely correlated with those in $C h d 2^{\mathrm{m}} / \mathrm{m} \mathrm{mEFs}$, where Chaserr was downregulated and $C h d 2$ was upregulated (Fig. $4 \mathrm{~d}$ and Supplementary Fig. 4b). We also profiled Chaserr ${ }^{-1-} C h d 2^{\mathrm{m} / \mathrm{m}}$ mEFs, and found no correlation between the changes observed between these and WT mEFs, and the changes in Chaserr ${ }^{-1-}$
mEFs (Spearman $R=-0.003, P=0.68$ ). Instead, changes in Chaserr ${ }^{-1}$ Chd $2^{\mathrm{m} / \mathrm{m}} \mathrm{mEFs}$ were similar to those in Chd $2^{\mathrm{m} / \mathrm{m}}$ mEFs (Supplementary Fig. 4b). The correlations between expression changes should be interpreted with caution as they are derived from different crosses, and yet they support the notion that the downstream effects of loss of Chaserr are driven by $\mathrm{CHD} 2$ upregulation.

Chaserr loss leads to transcriptional interference between close genes. Inspection of the loci of some of the most downregulated genes in Chaserr ${ }^{-/}$mEFs, such as Ctsk and Dapk3 (Fig. 4c), led us to suspect that promoters of some of the downregulated genes might be preferentially found in close proximity to transcription 
a

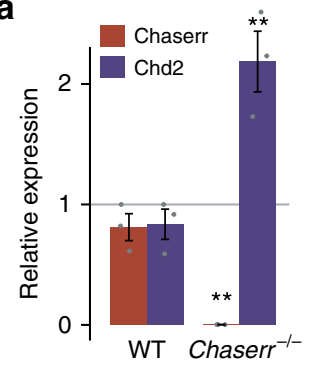

b

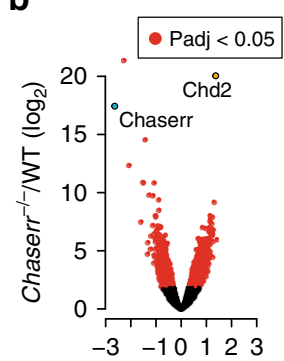

C

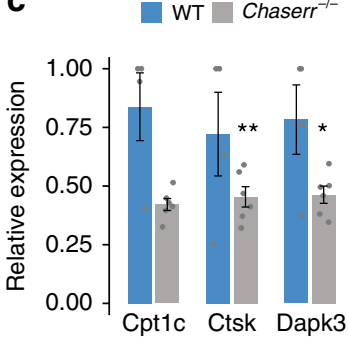

d

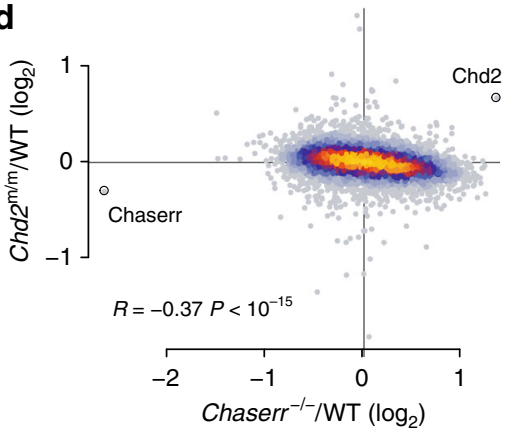

$\mathbf{f}$

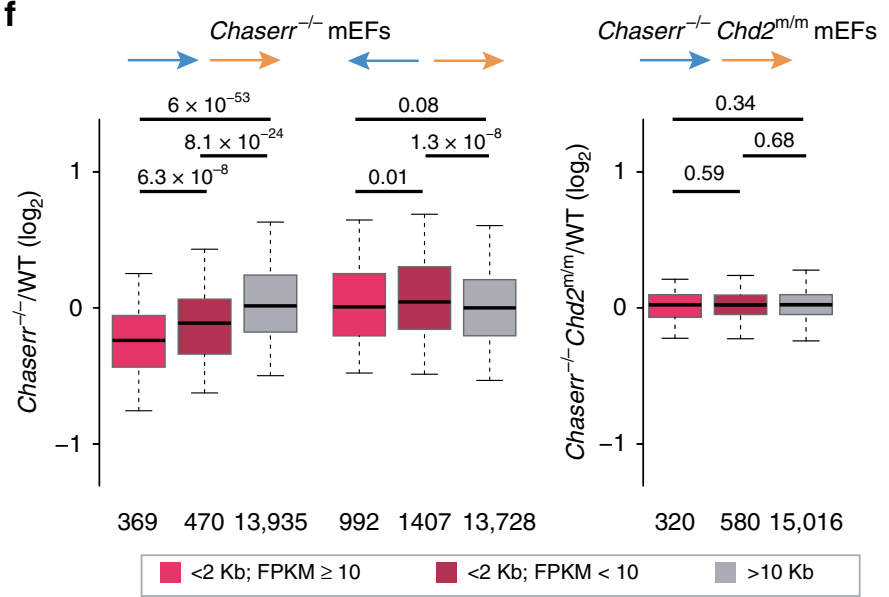

e

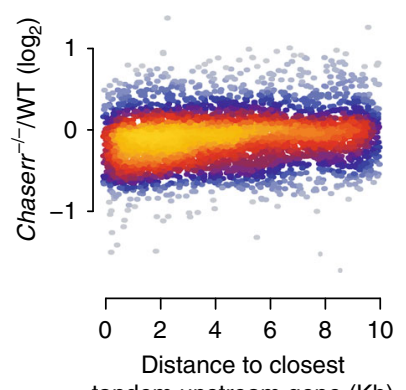

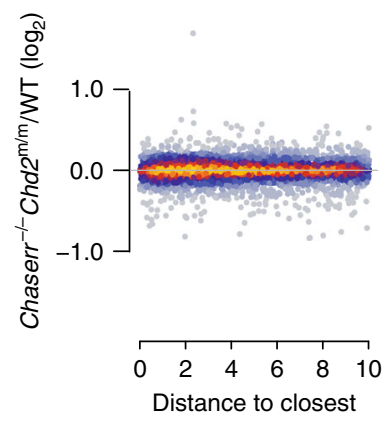

tandem upstream gene $(\mathrm{Kb})$ tandem upstream gene $(\mathrm{Kb})$

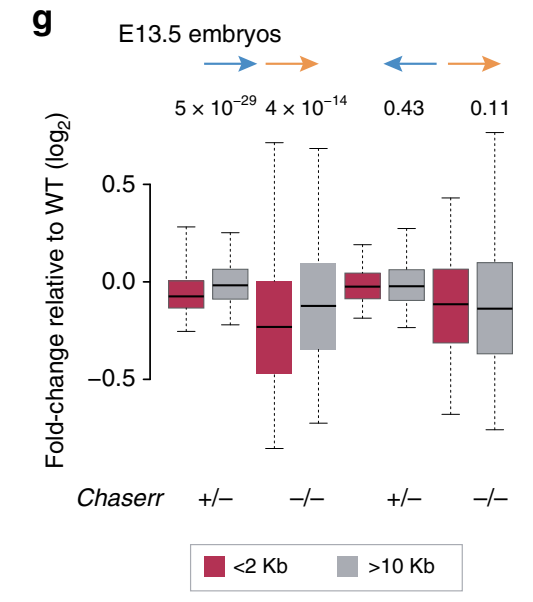

Fig. 4 Transcriptional dysregulation following loss of Chaserr. a qRT-PCR (left) and Western blot (right) of Chd2 expression in mEFs from the indicated background. $n=3$. qRT-PCR normalized to Actb. Western blot shows mEFs from three embryos for each background. Error bars show S.E.M. ${ }^{\star \star} P<0.01$ (two-sided $t$ test). b Volcano plots for the indicated differential expression comparison by using DESeq262. $\mathbf{c}$ qRT-PCR of the indicated genes in WT and Chaserr $^{-/-} \mathrm{mEFs}$. Normalized to Actb. WT $n=4$, Chaserr ${ }^{-/-} n=6{ }^{\star} P<0.05,{ }^{\star \star} P<0.01$ (two-sided $t$ test). d Changes in gene expression in Chaserr ${ }^{-/-}$ and $C h d 2^{\mathrm{m}} / \mathrm{m} \mathrm{mEFs}$. Correlation computed by using Spearman's correlation. Color indicates point density. e Change in expression in Chaserr ${ }^{-/-}$or Chaserr ${ }^{-/-}$Chd $2^{\mathrm{m} / \mathrm{m}}$ vs. WT mEFs and distance to the $3^{\prime}$ end of the closest upstream gene transcribed from the same strand, for genes for which this distance is $<10 \mathrm{~kb}$. Color indicates point density. $\mathbf{f}$ Changes in gene expression in Chaserr ${ }^{-/-}$or Chaserr ${ }^{-/-}$Chd2 ${ }^{\mathrm{m}} / \mathrm{m}$ vs. WT mEFs for genes with the indicated distances from the closest upstream gene. Genes with distance $<2 \mathrm{~kb}$ were further subdivided based on the expression levels of the upstream gene. The number of genes in each group is indicated below the distance threshold. Boxplots show the 5th, 25th, 50th, 75th, and 95th percentiles. $\mathbf{g}$ As in f, for the E13.5 whole embryo RNA-seq. P-values computed by using Wilcoxon rank-sum test. Ratio quantifications are shown below Western blots. Source data are provided as a Source Data file. Boxplots show the 5th, 25th, 50th, 75th, and 95th percentiles

termination sites (TTSs) of genes transcribed on the same strand (and thus potentially susceptible to transcriptional interference), in a similar organization to the Chaserr-Chd2 locus. Indeed, we found a mild yet highly significant correlation between changes in gene expression in Chaserr ${ }^{-1}$ mEFs and the distance to the TTS of the closest tandem upstream gene (Spearman $R=0.2, P<2 \times$ $10^{-15}$ ), with the effect observed mostly when the distance was shorter than $\sim 6 \mathrm{~kb}$ (Fig. 4e). In contrast, no such effect was observed in Chaserr $^{-1-}$ Chd $2^{\mathrm{m} / \mathrm{m}}$ mEFs $(R=0.0055, P=0.45$, Fig. 4e). Further, the downregulation was stronger when the expression of those upstream neighboring genes was higher
(Spearman $R=-0.17, P=3.4 \times 10^{-7}$ between the change in expression of the downstream gene and the absolute expression of the upstream gene for intergenic distances $<2 \mathrm{~kb}$ ). Genes with a close and abundant upstream neighbor were significantly repressed in Chaserr ${ }^{-/}$mEFs, and in stark contrast, much smaller differences, and in the opposite direction, were observed for neighboring genes transcribed on opposite strands, when considering distances between $5^{\prime}$ ends (Fig. 4f). Similar trends were also found in Chaserr ${ }^{+/}$and Chaserr ${ }^{-l-}$ E13.5 embryos (Fig. 4g).

To further characterize the regulatory dysregulation, we performed ATAC-seq ${ }^{32}$ on WT and Chaserr ${ }^{-1}$ mEFs. 

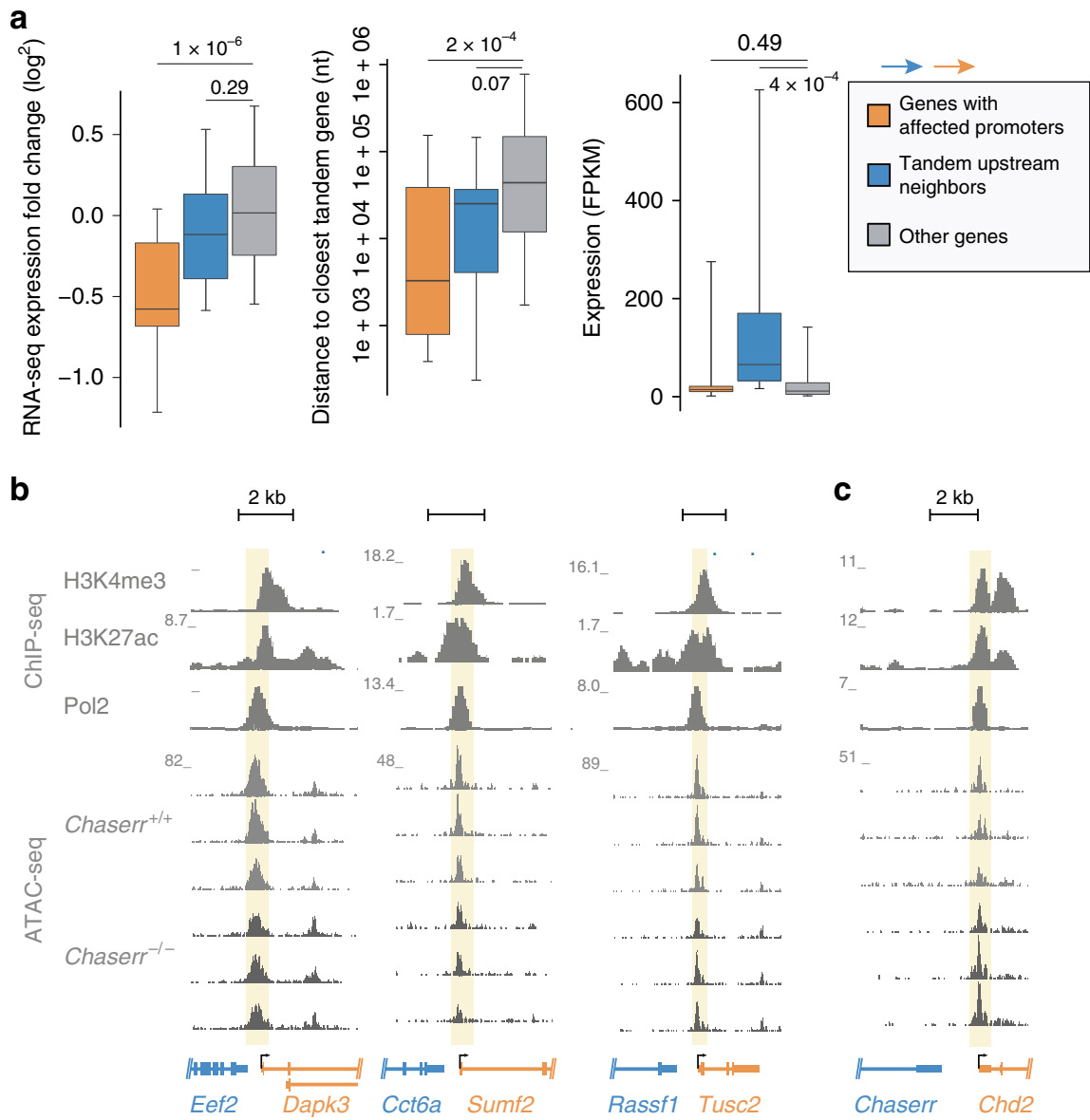

d

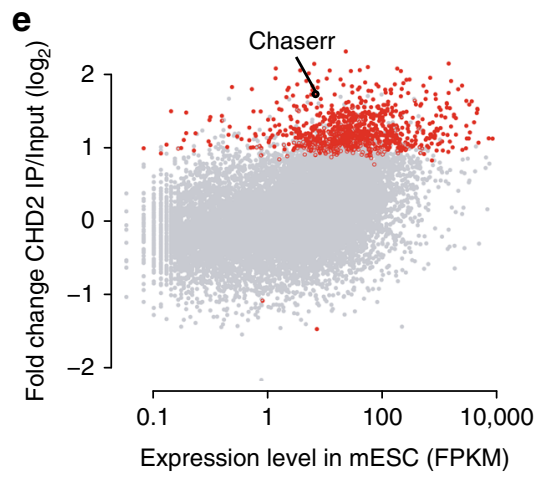

Fig. 5 Changes in chromatin accessibility following loss of Chaserr. a Changes in gene expression in Chaserr ${ }^{-/-} \mathrm{mEFs}$, distance to the closest upstream gene transcribed from the same strand, and the expression levels, for genes assigned to promoter peaks with reduced accessibility in Chaserr ${ }^{-/}-\mathrm{mEFs}_{\text {, }}$ their upstream neighbors on the same strand, and all other genes. Only genes with ATAC-seq peaks at their promoters were considered. P-values computed with two-sided Wilcoxon rank-sum test. Boxplots show the 5th, 25th, 50th, 75th, and 95th percentiles. $\mathbf{b}$ Examples of loci of genes with reduced accessibility in their core promoters in the Chaserr-/- background. Top: ChIP-seq coverage in mEFs from the ENCODE project. Bottom: read coverage in ATAC-seq data from mEFs isolated from three embryos from each background. $\mathbf{c}$ As in b, for the Chaserr/Chd2 locus. d Metagene of CHD2 occupancy ${ }^{10}$ on genes in mESCs, divided into four groups based on their expression FPKM. e Enrichment of CHD2 occupancy in mESCs in the 2-kb region downstream of the TTS when compared with input, as a function of mESC gene expression levels. Genes with adjusted $P<0.05$ are in red. Source data are provided as a Source Data file

Consistently with the increase in $C h d 2$ production, we observed a mild increase of $\sim 7 \%$ in accessibility of the Chd2 promoter in Chaserr $^{-1-}$ mEFs. Comparing WT and Chaserr ${ }^{-/-} \mathrm{mEFs}$, we found 80 and 677 regions with increased or reduced accessibility, respectively (DESeq $2<0.05$ ), with 6 induced and 24 reduced peaks occurring near TSSs. Promoter peaks whose accessibility was significantly decreased in Chaserr ${ }^{-/}$mEFs were associated with repressed genes in the RNA-seq data (Fig. 5a). Strikingly, these promoters were separated by short intergenic regions from TTSs of other genes on the same strand (Fig. 5a, b, median distance of $3.3 \mathrm{~kb}$ compared with $43.8 \mathrm{~kb}$ for unaffected promoters). Notably, the upstream genes were expressed at dramatically higher levels than the affected genes or other genes (17-fold difference between median expression, Fig. 5a), and their expression was not substantially affected by loss of Chaserr (Fig. 5a). Promoters of 96 additional genes that showed reduction 
of $>25 \%$ in Chaserr $^{-1-}$ mEFs in the RNA-seq data and had an upstream neighbor on the same strand within $<2 \mathrm{~kb}$, had reduced ATAC-seq signal in Chaserr ${ }^{-1}$ mEFs when considered as a group $(P=0.014$, Wilcoxon two-sided test). Altogether, transcriptional interference from highly expressed and close upstream neighbors reduces promoter accessibility and gene expression of over 100 genes in Chaserr ${ }^{-1-}$ mEFs.

To test if these effects stem from CHD2 overexpression, we used the same methodology to compare Chaserr-l- Chd $2^{\mathrm{m} / \mathrm{m}}$ mEFs with WT mEFs, and observed far fewer changes in accessibility (20 regions with decreased accessibility and 64 with increased accessibility, based on the same criteria as above, and only 3 decreased peaks in promoter regions), further supporting the notion that the transcriptional interference in Chaserr ${ }^{-/}$cells is driven by excess CHD2 levels.

Interestingly, the presence of a TTS closely upstream of the promoters most affected by loss of Chaserr (Fig. 5a, b) resembles the organization of the Chaserr and Chd2 locus (Fig. 5c). By using tagged CHD2 MNase-ChIP-seq data from mESCs ${ }^{10}$, we observed significant occupancy of CHD2 protein in the regions downstream of TTSs of hundreds of highly expressed genes, including downstream of Chaserr TTS (Fig. 5d, e), suggesting that the effect seen on promoter accessibility and expression of genes found closely downstream of TTSs of highly expressed genes might be a direct result of increased CHD2 activity at intergenic regions. In summary, CHD2 appears to function in regions downstream of TTS in WT cells, particularly at highly expressed genes, and the CHD2 hyperactivity caused by loss of Chaserr leads to repression of promoters found immediately downstream to those regions.

Chaserr loss leads to reduction in Pol2 pausing. An additional underlying factor contributing to dysregulation of gene expression emerged from ChIP-seq ${ }^{33}$ analysis of Pol2 in Chaserr ${ }^{-/-}$and WT mEFs. We observed a significant correlation between changes in gene expression and changes in Pol2 occupancy at gene bodies, but not at promoters (Fig. 6a, b). This observation, together with the known roles of CHD1 in regulation of Pol2 pausing ${ }^{34}$, led us to suspect that Pol2 pausing might be affected by the increase in CHD2 levels caused by Chaserr loss. Indeed, genes upregulated in Chaserr $^{-/-} \mathrm{mEFs}$ were associated with a substantially higher pausing index in WT mEFs in our Pol2 ChIP-seq data, as well as in $\mathrm{mEF}$ s profiled by the ENCODE project (Fig. 6c); generally, increases in gene expression were correlated with reduction in Pol2 pausing at the affected genes (Fig. 6d). Overall, 400 (15.3\%) of the genes significantly dysregulated in Chaserr-l- mEFs were associated with a corresponding change of $>25 \%$ in $\mathrm{Pol} 2$ pausing.

CHD2 binds nascent RNAs and promotes gene expression. Several recent studies identified CHD2 as a noncanonical RNAbinding protein ${ }^{35-37}$. We therefore tested whether CHD2 might associate with Chaserr and with some of the RNAs deregulated by loss of Chaserr or of Chd2. Using RNA immunoprecipitation $(\mathrm{RIP})^{38}$ followed by rRNA-depleted RNA sequencing (RIP-seq, see Methods), we found that CHD2 significantly associates with 2,427 RNAs, and that Chaserr is one of the most enriched RNAs in the IP compared with either the input or IgG controls (Fig. 6e and Supplementary Fig. 4c). Interestingly, CHD2 also substantially binds its own pre-mRNA (Fig. 6e). CHD2 RIP libraries contained a large number of intronic reads in the Chaserr locus (Fig. 6g) and in other loci (Fig. 6h and Supplementary Fig. 4d), suggesting that CHD2 predominantly binds nascent RNA. Strikingly, CHD2-bound RNAs were significantly upregulated in Chaserr $^{-l-} \mathrm{mEFs}$, and conversely repressed relative to other genes in $C h d 2^{\mathrm{m} / \mathrm{m}}$ or Chaserr ${ }^{-1-}$ Chd $d^{\mathrm{m} / \mathrm{m}} \mathrm{mEFs}$ (Fig. 6f). These data suggest that RNA binding by $\mathrm{CHD} 2$ typically promotes gene expression, including expression of the CHD2-bound Chaserr (which is repressed in $C h d 2^{\mathrm{m} / \mathrm{m}} \mathrm{mEFs}$, Fig. $4 \mathrm{~d}$ ), and thus Chaserr expression can act as a sensor of CHD2 levels.

\section{Discussion}

Decoding lncRNA functions is a formidable challenge, in particular because of the substantial heterogeneity in their biology and modes of action. A prominent group of vertebrate lncRNAs, including some of the most conserved ones, are found in regions flanking genes involved in transcription, including numerous chromatin modifiers. We hypothesized that such co-localization may imply a connection between the biology of the chromatin modifier and the mode of action of the lncRNA, potentially through a feedback loop. By focusing on one such pair that is particularly highly conserved in evolution, we uncovered a lncRNA-mediated circuit that regulates the expression of Chd2.

Negative autoregulatory feedback loops are prevalent in RNA biology, and help tune levels of factors involved in mRNA splicing, polyadenylation, editing, and modifications, and in the processing of small RNAs ${ }^{39-45}$. There are also examples of such feedback loops regulating transcription-related genes ${ }^{46}$; however to the best of our knowledge, there are no known autoregulatory loops involving chromatin modifiers in mammals. We propose a model in which the Chaserr-Chd 2 tandem organization forms a negative feedback loop that tunes CHD2 levels. The genomic occupancy of CHD2 in regions immediately downstream of TTSs (Fig. 5d, e), combined with the correlation between changes in gene expression and changes in Pol2 occupancy in gene bodies in Chaserr $^{-/-}$mEFs (Fig. 6b), suggests that CHD2 acts at least partly within transcribed regions and in TTS-proximal regions. Accordingly, we show that under conditions of excess levels of CHD2 protein, this TTS-proximal occupancy is associated with transcriptional interference on downstream neighbors of highly expressed genes, especially when the intergenic regions between them are particularly short (Figs. $4 \mathrm{e}-\mathrm{g}, 5 \mathrm{a}, \mathrm{b}$ ). Due to the short intergenic region between Chaserr and Chd2, we suggest that such transcriptional interference serves as the basis of a negative feedback loop that leads to repression of $C h d 2$ production when $\mathrm{CHD} 2$ protein levels are high, thus maintaining a tight control on its expression. In cells with Chaserr loss of function, this loop is compromised, and CHD2 is upregulated. Supporting this model, in Chaserr $^{+/-}$Chd $2^{\mathrm{m} /+}$ tissues, increase in CHD2 protein leads to repression of transcription of the $C h d 2^{\mathrm{m}}$ allele, which is found next to an intact Chaserr (Fig. 3d). Furthermore, reduction in CHD2 protein in $C h d 2^{\mathrm{m} / \mathrm{m}} \mathrm{mEF}$ leads to a decrease in Chaserr levels and an increase in Chd 2 mRNA levels (Fig. 4d). Last, we found that $\mathrm{CHD} 2$ protein associates with the Chaserr RNA (Fig. 6e), supporting the possibility that excess CHD2 levels can be sensed by the Chaserr locus.

The exact mechanism by which Chaserr represses Chd2 expression remains unknown. The simplest feedback model suggests that Chaserr transcription, or perhaps its termination and cleavage and polyadenylation, are important. Such a mechanism would resemble the activity of the SRG1 noncoding RNA in the yeast $S$. cerevisiae that represses the transcription of SER3, which is found immediately downstream of SRG1 and on the same strand, by deposition of nucleosomes at the SER3 promoter that prevent binding of transcription activators ${ }^{47-51}$. The genomic arrangement of SRG1 and SER3 resembles that of Chaserr and Chd2, though the $3^{\prime}$ end of SRG1 overlaps SER3 promoter ${ }^{48}$, and the distance between their promoters is much shorter ( $~ 500$ bp between SRG1 TSS and SER3 TSS vs. $\sim 17 \mathrm{~kb}$ between the TSS of Chaserr and TSS of Chd2).

Another possibility is that the promoters of Chaserr and of Chd2 compete for binding to shared enhancer elements, and 
a

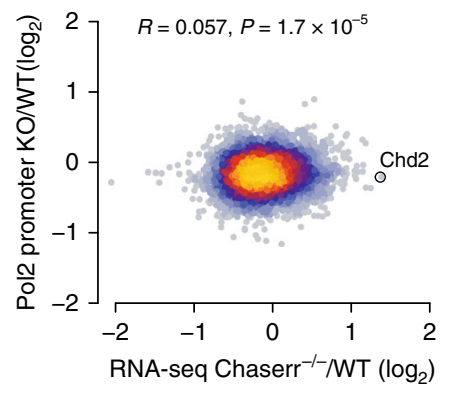

C

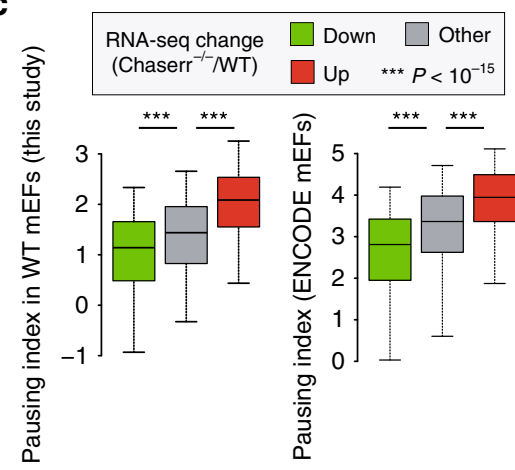

e

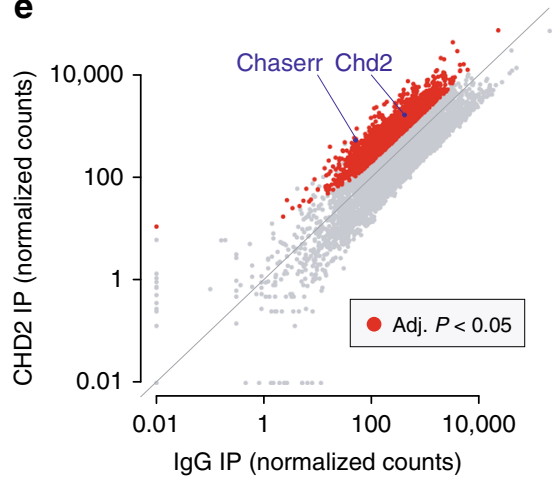

9

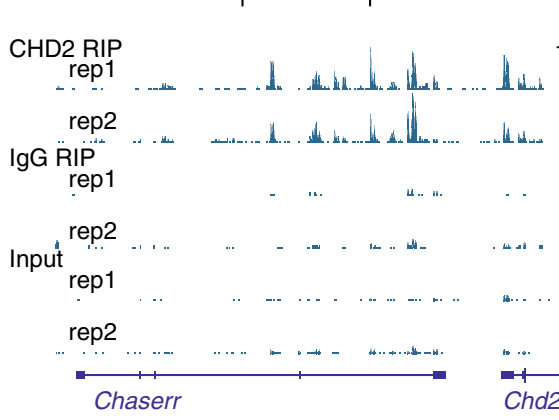

b

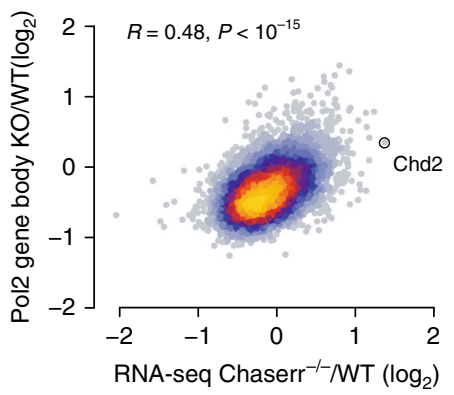

d

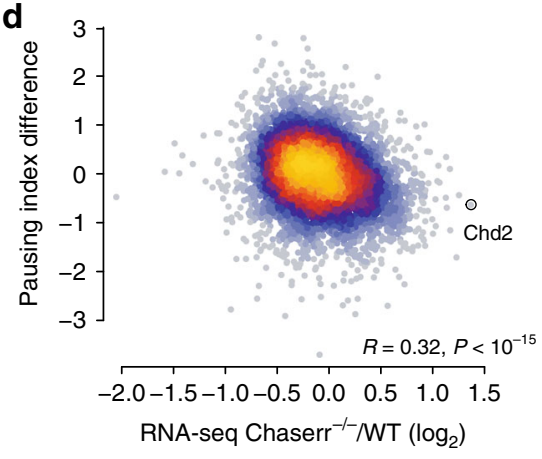

$\mathbf{f}$

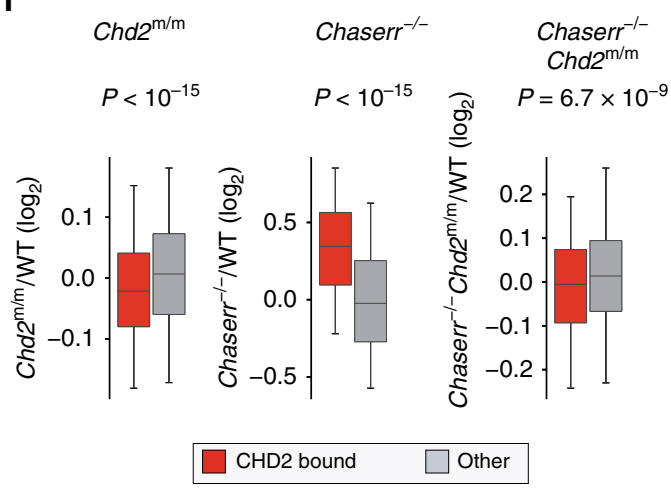

$\mathbf{h}$

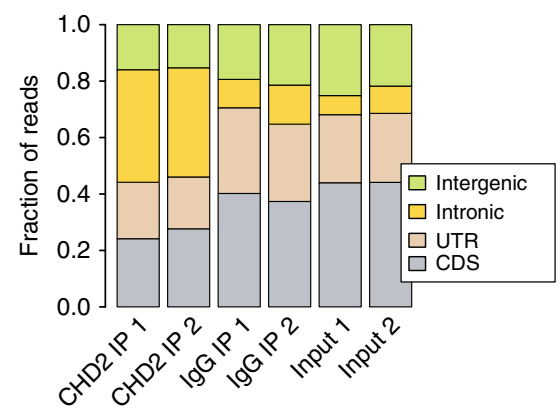

Fig. 6 Changes in Pol2 occupancy in Chaserr $/-$ mEFs and RNA binding by CHD2. a Correlation between changes in gene expression levels and changes in Pol2 occupancy at the promoter region ( \pm 300 bases around TSS). Color indicates point density. $\mathbf{b}$ As in $\mathbf{a}$, but for changes in Pol 2 occupancy at gene body regions (defined from $1 \mathrm{~kb}$ downstream of the TSS to the TTS). c Pol2 pausing index (see the "Methods" section) in our WT mEF Pol2 ChIP-seq data (left)

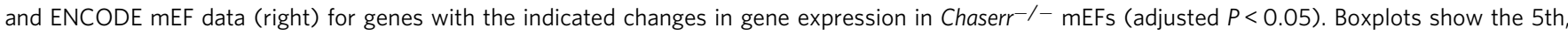
25th, 50th, 75th, and 95th percentiles. d Changes in Pol2 pausing index as a function of changes in gene expression between Chaserr ${ }^{-1-}$ and WT mEFs. Color indicates point density. e Normalized RIP-seq read counts within gene bodies in CHD2 and IgG RIP libraries (average of two replicates). Red dots correspond to genes with adjusted $P<0.05$ (DESeq2 analysis). $\mathbf{f}$ Changes in gene expression of genes significantly associated with $\mathrm{CHD} 2$ (from e) and other genes expressed in mEFs in the indicated RNA-seq-based comparison. Boxplots show the 5th, 25th, 50th, 75th, and 95th percentiles. $\mathbf{g}$ RIP-seq read coverage in the Chaserr locus. $\mathbf{h}$ Fractions of reads mapping to the indicated regions in the indicated RIP-seq library. Source data are provided as a Source Data file 
elimination of the Chaserr promoter increases the ability of these enhancers to boost Chd2 expression, as recently described for $M Y C$ and $P V T 1^{52}$. Both Chaserr and Chd2 appear to be under the shared control of several enhancer regions found in the $\sim 200-\mathrm{kb}$ gene desert upstream of Chaserr (Fig. 2g and Supplementary Fig. 5), and those enhancers potentially regulate both genes together, which can explain the tight co-expression between Chaserr and Chd2 (Fig. 1c). Indeed, deletion of the Chaserr promoter in mESCs leads to an increase in contacts between the Chd2 promoter and three broad upstream enhancer regions (Fig. 2g). Enhancer competition may therefore contribute to the increase in Chd2 expression in Chaserr ${ }^{-1-}$ cells. However, it is unlikely that such competition explains much of the CHD2 upregulation, as we observe similar levels of upregulation when using ASO Gapmers in different cellular systems (Fig. 2e, Fig. 3e, Supplementary Fig. 2b, c, and see below). In addition, removing just the gene body of Chaserr, which leaves the promoter intact, does not substantially affect Pol2 occupancy at the promoter (Supplementary Fig. 2a), nor the landscape of spatial contacts between the Chd2 promoter and the upstream enhancers (Fig. $2 \mathrm{~g}$ ), despite leading to a similar upregulation of $C h d 2$ levels (Fig. 2e).

There is evidence that the RNA product of Chaserr, and not just its intact locus or the act of its production, are important for Chd2 repression. The exon-intron architecture and sequence of Chaserr are highly conserved, suggesting that production of a particular RNA species, or splicing at particular locations, is important either because of the RNA product itself, or because they modulate the amount of transcription or Pol2 velocity upon encounter with the $\sim 2$-kb intergenic region between Chaserr and Chd2. The similar effects we observed when deleting Chaserr promoter or gene body, or when targeting the RNA with ASOs or Gapmers, also point to the importance of the RNA product, though we cannot rule out that ASOs and Gapmers may affect transcription by cleaving the nascent transcript.

Interestingly, in contrast to previous reports based on a gene trap inserted in intron 27 of Chd $2^{14,53,54}$, nearly complete loss of CHD2 protein appears to be largely compatible with normal viability in mice in our hands, and in the similar mouse model generated by the EUCOMM consortium ${ }^{15}$. The previous observations of embryonic lethality following CHD2 loss of function can potentially be attributed to a dominant negative effect of the truncated protein product created by the gene trap. In contrast to the CHD2 loss models, we report here that increase in CHD2 levels brought about by loss of even a single copy of Chaserr is toxic and leads to perinatal lethality. We note that this is one of the most severe phenotypes reported so far for loss of a lncRNA ${ }^{6,55}$. The severe, CHD2-mediated phenotype is consistent with the high conservation of Chaserr-Chd2 genomic organization and sequence during 500 million years of vertebrate evolution. Of the lncRNAs annotated in human and mouse, only $~ 5 \%$ ( $\sim 100$ genes) have evidence of conservation in fish ${ }^{26,56}$, and we were able to identify homologs of Chaserr in every vertebrate species we examined (Fig. 1a).

Our results have important implications from the therapeutic perspective. Individuals that bear mutations in the $\mathrm{CHD} 2$ gene exhibit epilepsy and neurodevelopmental disorders ${ }^{16}$. In all described cases, these individuals are haploinsufficient for $\mathrm{CHD} 2$, and so bear an intact WT copy of CHD2. Therefore, increase of CHD2 expression through perturbation of Chaserr, e.g., by using antisense oligonucleotides, might have a therapeutic benefit. Importantly, Chaserr is highly conserved between human and mouse (Fig. 1a), and targeting of the human CHASERR by using Gapmers in human MCF7 and SH-SY5Y cells leads to an increase in CHD2 mRNA and protein levels (Supplementary Fig. 6), suggesting that the results we observe in the mouse model are of direct relevance to human CHASERR.
Similarly to many other chromatin-remodeling factors that are increasingly implicated in human disease, the precise molecular function of $C h d 2$ and the direct consequences of its dysregulation are poorly understood. Here, we could leverage the similarity between the genomic arrangements of Chaserr and Chd2 and those of the genes most affected by Chaserr perturbation to highlight the potential role of chromatin remodeling that CHD2 plays downstream of TTSs. Decoding lncRNA functions can therefore provide important insight into other layers of gene regulation. As chromatin modifiers are often flanked by lncRNAs, some as highly conserved as Chaserr, we expect that further research into the functions of these lncRNAs may uncover additional paradigms in chromatin biology.

\section{Methods}

Animals. The study was conducted in accordance with the guidelines of the Weizmann Institutional Animal Care and Use Committee (IACUC) and approved by it. C57black6 Ola HSD mice were purchased from Harlan Laboratories (Rehovot, Israel). All other mouse strains were bred and maintained at the Veterinary Resources Department of the Weizmann Institute.

Generation of Chaserr ${ }^{+/-}$mice. The Chaserr $^{+/-}$mice were generated as in Wang et al. ${ }^{24}$. All mice were generated by standard procedures at the Weizmann transgenic core facility. We used four single-guide RNAs (sgRNAs 1-4, see Supplementary Data 2), two targeting regions before the Chaserr transcription start site and two targeting regions in the first intron, which resulted in two founder lines. Chaserr mutant mice were genotyped by using primers flanking the targeted region followed by Sanger sequencing. To generate Chd 2 mutant mice we used one sgRNA targeted to the third exon of Chd 2 mRNA. Chd 2 mutant mice were genotyped by amplicon sequencing that identified a founder with a frameshift in the main ORF. Amplicon library preparation was as follows: Chd2 mutant locus was amplified by using Chd2 genotype nesting primers (Supplementary Data 2). Thereafter $1 \mu$ of PCR reaction was used as template for the addition of R1 and R2 Illumina adaptors (R1/R2_Exon3_Chd2, Supplementary Data 2), followed by fragment AMPure (Beckman Coulter, A63881) size selection and cleanup. A second PCR reaction was used in order to add sequencing barcodes to the amplicons, followed by AMPure size selection. Indexed amplicons were pooled and sequenced on NextSeq 500. To genotype $C h d 2^{m}$ lines routinely, we used PCR with primers flanking the mutated region and digested the PCR product with the DdeI restriction enzyme whose recognition sequence is compromised in the mutated DNA. The fragment sizes were then analyzed on a $2 \%$ agarose gel. To generate Chaserr ${ }^{-/-}$Chd $2^{\mathrm{m} / \mathrm{m}}$ mice, we used $C h d 2^{\mathrm{m} / \mathrm{m}}$ background and continued as described above for generation of the Chaserr ${ }^{-}$allele, by using only two sgRNAs (sgRNA2+3, Supplementary Data 2). sgRNA injection was done on CB6F1 Ola HSD mice that were later backcrossed with C57BL/6 Ola HSD. All the experiments were done on 4- to 15-week-old mice from F2 to F5 generations and E9.5-E18.5 developmental time points.

Tissue culture. R1 mESCs (kind gift from the Nagy lab) were routinely cultured in mouse ES medium (mESM) consisting of $500 \mathrm{ml}$ of DMEM (Gibco, 11965-092), $15 \%$ ES-grade Fetal Calf Serum (Biological Industries), sodium pyruvate $1 \mathrm{mM}$ (Gibco, 11360-039), nonessential amino acids $1 \times($ Gibco, 11140-035), $0.1 \mathrm{mM}$ bmercaptoethanol (Sigma, M6250-250ML), penicillin-streptomycin (Biological Industries), and $1000 \mathrm{U} / \mathrm{ml} \mathrm{LIF} \mathrm{(Weizmann} \mathrm{Proteomics} \mathrm{Unit).}$

All other cell lines (from ATCC) were routinely cultured in DMEM containing $10 \%$ fetal bovine serum and $100 \mathrm{U}$ penicillin $/ 0.1 \mathrm{mg} \mathrm{ml}^{-1}$ streptomycin at $37^{\circ} \mathrm{C}$ in a humidified incubator with $5 \% \mathrm{CO}_{2}$. Cell lines were routinely tested for mycoplasma contamination and were not authenticated.

Primary mEFs were isolated from E13.5 embryo. Heads were used to genotype the embryo, while the rest of the embryo (liver excluded) was dissociated with $1 \mathrm{~mL}$ of trypsin $0.05 \%$ in an Eppendorf tube for $5 \mathrm{~min}$ at $37^{\circ} \mathrm{C}$, pipetted for complete dissociation, and incubated for another $5 \mathrm{~min}$ at $37^{\circ} \mathrm{C}$. Cells were then supplemented with $2 \mathrm{~mL}$ of medium, centrifuged for $5 \mathrm{~min}$ at $192 \times g$. Cells were seeded on $10-\mathrm{cm}$ gelatin-coated $(0.1 \%)$ plates $^{57}$.

Neuronal differentiation. Neuronal differentiation was performed as previously described $^{58}$. mESCs were first grown in the absence of mEFs for two passages and then seeded on gelatin-coated plates at a density of $1.5 \times 10^{5}$ in N2B27 medium: 1:1 mixture of DMEM/F12 (Sigma) supplemented with N2 (Gibco), and Neurobasal medium (Gibco) supplemented with B27 (Gibco), 1× Glutamax (Gibco), $0.1 \mathrm{mM} \beta$ mercaptoethanol (Sigma), $100 \mathrm{U} / \mathrm{ml}$ penicillin, and $0.1 \mathrm{mg} / \mathrm{ml}$ streptomycin (Biological industries). After 4 days under these conditions, $3 \times 10^{5}$ cells were replated on Poly-D-Lysine (Sigma, P6407) and Laminin (Life, 23017-015) coated plates, in N2B27 medium supplemented with $20 \mathrm{ng} / \mathrm{ml}$ FGF2 (Peprotech, 100-18B-50/10018B-100). After $24 \mathrm{hr}$, FGF2 was removed and cells were cultured for 3 additional days $^{58}$. 
Transfections. mESCs were transfected with electroporation of the Lonza protocol (http://bio.lonza.com/fileadmin/groups/marketing/Downloads/Protocols/ Generated/Optimized_Protocol_309.pdf). HEK293T cells were transfected by using PolyEthylene Imine (PEI ${ }^{59}$ (PEI linear, $\mathrm{M} r 25,000$, Polyscience). Neuro2A transfection: $2 \times 10^{5}$ cells were seeded in a six-well plate and transfected by using Lipofectamine 3000 (Life Technologies, L3000-008) with LNA1, LNA2, or a mix of LNA1-4 or with ASO1, ASO2, ASO3, or a mix of ASO1-3 to a final concentration of $50 \mathrm{nM}$. For transfection of MCF7 cells, $2 \times 10^{5}$ cells were seeded in a six-well plate and transfected by using PEI with LNA h1 and/or LNA h2 to a final concentration of $50 \mathrm{nM}$. For transfection of SH-SY5Y cells, $2 \times 10^{5}$ cells were seeded in a six-well plate and transfected by using DharmaFECT 4 Transfection Reagent (Dharmacon, T-2002-03) following the manufacturer's protocol with LNA h1 and/ or LNA h2 to a final concentration of $50 \mathrm{nM}$. Endpoints for all knockdown experiments were at $48 \mathrm{hr}$ post transfection.

Genome editing in mESCs. To generate Chaserr $\mathrm{p} / \mathrm{p}$ mESCs, $2 \times 10^{6} \mathrm{mEF}$-depleted cells were transfected with sgRNAs $2+3$ and pCas9_GFP (a gift from Kiran Musunuru, Addgene \#44719). To generate Chaserr $\mathrm{b} / \overline{\mathrm{b}} \mathrm{mESCs}, 2 \times 10^{6}$ cells were transfected with sgRNAs $2+5$ and pCas9_GFP. The next day, fresh mESC medium was supplemented with $1 \mu \mathrm{g} / \mathrm{ml}$ puromycin (Invivogen, ant-pr-1) for $72 \mathrm{hr}$, while replacing medium every $24 \mathrm{hr}$. Next, $3-4 \times 10^{3}$ cells were seeded at low density on a $10-\mathrm{cm}$ plate until single colonies formed; thereafter, colonies were picked, expanded, genomic deletion was verified by PCR sequencing, and expression was tested by RT-qPCR and RNA-seq.

RNA and RT-qPCR. Total RNA was extracted from different cell lines and mouse tissues, by using TRIREAGENT (MRC) according to the manufacturer's protocol. cDNA was synthesized by using qScript Flex cDNA synthesis kit (95049, Quanta). Fast SYBR Green master mix (4385614) was used for qPCR.

RNA-seq. Strand-specific mRNA-seq libraries were prepared from 500 to $4000 \mathrm{ng}$ of total RNA by using the TruSeq Stranded mRNA (Illumina) or SENSE mRNASeq (Lexogen) library preparation kits, according to the manufacturer's protocols. Libraries were sequenced on a NextSeq 500 to obtain 38-50-nt paired-end reads. Coverage tracks for the UCSC genome browser were prepared by aligning reads to the $\mathrm{mm} 9$ genome assembly with $\mathrm{STAR}^{60}$. Gene expression levels were quantified with RSEM ${ }^{61}$ and a RefSeq gene annotation database that was manually edited to correct the annotation of the last exon of Chaserr. Differential expression was computed with DESeq2 with default settings ${ }^{62}$. Genomic context was also analyzed by using the RefSeq gene annotations. RNA-seq and ATAC-seq datasets are deposited in GEO database under the accession GSE124375. RNA-seq data from previous studies were downloaded from the SRA database, and quantified with RSEM with the same annotation file.

Western blot. Total protein was extracted from tissues and cell lines by lysis with RIPA supplemented with protease inhibitors and DTT $1 \mathrm{mM}$. Proteins were resolved on $8-10 \%$ SDS-PAGE gels and transferred to a polyvinylidene difluoride (PVDF) membrane. After blocking with 5\% nonfat milk in PBS with 0.1\% Tween20 (PBST), the membranes were incubated with the primary antibody followed by the secondary antibody conjugated with horseradish peroxidase. Blots were quantified with Image Lab software. Primary antibodies were as follows: anti-Chd2 (Millipore, \#MABE873, 1:1,000 dilution), anti- $\beta$-tubulin (Sigma, \#T4026, 1:2,000 dilution). Secondary antibodies were as follows: anti-rat (\#AP136P, 1:10,000 dilution), anti-mouse (\#115-035-003, 1:10,000 dilution).

ChIP-seq. In total, $1 \times 10^{7} \mathrm{mEFs}$ were cross-linked with formaldehyde at $1 \%$ final concentration, for $10 \mathrm{~min}$ at room temperature, and then quenched with $125 \mathrm{mM}$ glycine for $5 \mathrm{~min}$ at room temperature. Cells were centrifuged for $5 \mathrm{~min}, 376 \times g$ at $4{ }^{\circ} \mathrm{C}$, and washed twice with PBS supplemented with PIC (protease inhibitor cocktail). Each pellet was then lysed in $1 \mathrm{~mL}$ of lysis buffer (5 mM PIPES, pH 8.0, $85 \mathrm{mM} \mathrm{KCl}$, Igepal $(10 \mu \mathrm{l} / \mathrm{ml})$, and PIC), incubated for $15 \mathrm{~min}$ at $4{ }^{\circ} \mathrm{C}$, and then centrifuged for $5 \mathrm{~min}$ at $21130 \times \mathrm{g}$ at $4^{\circ} \mathrm{C}$. Pellets were resuspended in $200 \mu \mathrm{l}$ of nuclei lysis buffer (50 mM Tris-Cl, $\mathrm{pH}$ 8.1, $10 \mathrm{mM}$ EDTA, $1 \%$ SDS, and PIC) for $30 \mathrm{~min}$ at $4{ }^{\circ} \mathrm{C}$. Lysates were then sonicated (Bioruptor, \#B01020001) for 12 cycles of $30 \mathrm{~s}$ ON, $30 \mathrm{~s}$ OFF. Pol2 antibody ( $5 \mu \mathrm{g}$, anti-Rbp1 NTD (D8L4Y), \#14958) was bound to $\mathrm{A} / \mathrm{G}$ magnetic beads in $1 \mathrm{~mL}$ of binding/washing buffer (PBS supplemented with $0.5 \%$ TWEEN and $0.5 \%$ BSA) for $1 \mathrm{hr}$ at room temperature. Chromatin lysate was then diluted $\times 9$ volume with IP dilution buffer $(50 \mathrm{mM}$ Tris- $\mathrm{HCl}$, $\mathrm{pH} 7.4,150 \mathrm{mM} \mathrm{NaCl}, 1 \%$ Igepal, $0.25 \%$ deoxycholic acid, and $1 \mathrm{mM}$ EDTA, $\mathrm{pH}$ 8.0); the coupled beads were washed and added to the chromatin lysate and left for slow rotation overnight at $4^{\circ} \mathrm{C}$. Beads were handled as described ${ }^{63}$. Briefly, beads were washed five times with RIPA buffer $(10 \mathrm{mM}$ Tris- $\mathrm{HCl}, \mathrm{pH}$ 8.0, $1 \mathrm{mM}$ EDTA, $\mathrm{pH} 8.0,140 \mathrm{mM} \mathrm{NaCl}, 1 \%$ Triton X-100, $0.1 \%$ SDS, and $0.1 \% \mathrm{Na}-\mathrm{DOC}$ ), twice with RIPA-500 buffer (10 mM Tris-HCl, pH 8.0, 1 mM EDTA, pH 8.0, $500 \mathrm{mM} \mathrm{NaCl}$, $1 \%$ Triton X-100, $0.1 \%$ SDS, and $0.1 \% \mathrm{Na}-\mathrm{DOC})$, twice with LiCl buffer ( $10 \mathrm{mM}$ Tris-HCl, pH 8.0, 1 mM EDTA, pH 8.0, $250 \mathrm{mM} \mathrm{LiCl,} 0.5 \% \mathrm{NP}-40$, and $0.5 \% \mathrm{Na}-$ DOC), and once with TE buffer ( $10 \mathrm{mM}$ Tris- $\mathrm{HCl}$, $\mathrm{pH} 8.0$ with $1 \mathrm{mM}$ EDTA, $\mathrm{pH}$ 8.0). Beads were eluted with direct elution buffer (10 mM Tris- $\mathrm{HCl}, \mathrm{pH} 8.0,5 \mathrm{mM}$ EDTA, $\mathrm{pH} 8.0,300 \mathrm{mM} \mathrm{NaCl}$, and $0.5 \% \mathrm{SDS}$ ) at room temperature. The eluate was incubated with RNaseA for $30 \mathrm{~min}$ at $37^{\circ} \mathrm{C}$, next with proteinase $\mathrm{K}$ for $2 \mathrm{hr}$ at $37^{\circ} \mathrm{C}$, and last overnight at $65^{\circ} \mathrm{C}$. DNA was purified with the Agencourt AMPure XP system (Beckman Coulter Genomics, A63881). Libraries were constructed as previously described ${ }^{63}$ and sequenced with paired-end sequencing on Illumina NextSeq 500

ATAC-seq. ATAC-seq was performed as previously described ${ }^{32}$. We isolated nuclei from $25 \times 10^{3} \mathrm{mEFs}$ derived from WT, Chaserr ${ }^{-1-}$, or Chaserr ${ }^{-1-}$ Chd $2^{\mathrm{m} / \mathrm{m}}$ backgrounds. Cells were centrifuged at $500 \mathrm{~g}$ for $5 \mathrm{~min}$, followed by a wash with $50 \mu \mathrm{l}$ of cold $1 \mathrm{x}$ PBS and centrifugation at $500 \times g$ for $5 \mathrm{~min}$. Cells were lysed with cold lysis buffer $\left(10 \mathrm{mM}\right.$ Tris-Cl, $\mathrm{pH} 7.4,10 \mathrm{mM} \mathrm{NaCl}, 3 \mathrm{mM} \mathrm{MgCl}_{2}$, and $0.1 \%$ IGEPAL CA-630). Immediately after lysis, nuclei were spun at $500 \times \mathrm{g}$ for $10 \mathrm{~min}$ at $4{ }^{\circ} \mathrm{C}$. Immediately following the nuclei prep, the pellet was resuspended in the transposase reaction mix $(12.5 \mu \mathrm{L}$ of $2 \times$ TD buffer, $1 \mu \mathrm{L}$ of Transposase (Illumina), and $11.5 \mu \mathrm{L}$ of nuclease-free water). The transposition reaction was carried out for $1 \mathrm{hr}$ at $37^{\circ} \mathrm{C}$. Directly following transposition, the reaction was treated with $2 \mu \mathrm{L}$ of $5 \% \mathrm{SDS}, 2 \mu \mathrm{L}$ of $20 \mathrm{mg} / \mathrm{ml}$ proteinase $\mathrm{K}$, and $5 \mu \mathrm{l}$ of cleanup buffer $\left(900 \mathrm{mM} \mathrm{MaCl}, 30 \mathrm{mM}\right.$ EDTA) for $30 \mathrm{~min}$ at $40^{\circ} \mathrm{C}$. The sample was purified by using $2 \times$ SPRI beads. Following purification, we amplified library fragments with $2 \times$ Kappa HiFi and $1.25 \mu \mathrm{M}$ of i5 and i7 primers (Illumina) using the following PCR conditions: $98^{\circ} \mathrm{C}$ for $2 \mathrm{~min}, 98^{\circ} \mathrm{C}$ for $20 \mathrm{~s}$, followed by thermocycling at $63^{\circ} \mathrm{C}$ for $30 \mathrm{~s}$ and $72{ }^{\circ} \mathrm{C}$ for $1 \mathrm{~min}$. We amplified the libraries for nine cycles. The libraries were purified by using $0.5 \times$ and $1.8 \times$ for double size selection cut off. Next we amplified the libraries by using a second PCR with the same conditions for five cycles. Lastly, the libraries were purified by using $2 \times$ SPRI beads. Libraries were sequenced with paired-end sequencing on Illumina NextSeq 500.

ATAC-seq and ChIP-seq data analysis. Reads were aligned to the $\mathrm{mm} 9$ genome assembly with Bowtie $2^{64}$, and ATAC-seq peaks were called by MACS2 ${ }^{65}$. Normalized read coverage files were computed by MACS2. ATAC-seq peaks from individual samples were merged with bedtools ${ }^{66}$. Read coverage was quantified by using Homer 4.9.1 ${ }^{67}$. Differential accessibility was computed with DESeq $2^{62}$ called by Homer ${ }^{67}$ using an annotation file based on RefSeq gene models, considering only genes longer than $2 \mathrm{~kb}$. Each gene was assigned with a single TSS based on FANTOM5 CAGE annotations for mEFs/mESCs, and with the most distal annotated TTS. The promoter region for each gene was defined as \pm 300 around that TSS, gene body as the region from $1 \mathrm{~kb}$ downstream of the TSS to the TTS, and the region downstream of the TTS as $2 \mathrm{~kb}$ from the TTS. ENCODE Pol2 ChIP-seq data (ENCFF001LOL accession) and CHD2 MNaseq-seq data were processed the same way. Pol2 pausing index was computed as the ratio between the Homernormalized average Pol2 occupancy in the promoter and the gene body (after adding a pseudocount of 0.5 to the total coverage in each region). Only genes with at least five normalized reads per $\mathrm{kb}$ in the gene body were considered.

Targeted chromosome conformation capture (4C). Following depletion from mEFs by 20 -min incubation on gelatin-coated plates, $3 \mathrm{C}$ was carried out on $5 \times 10^{6}$ mESCs essentially as described ${ }^{68}$, with the following slight modification: permeabilization buffer constitution was $10 \mathrm{mM}$ Tris- $\mathrm{HCl}, \mathrm{pH} 8,10 \mathrm{mM} \mathrm{NaCl}$, and $0.5 \%$ NP-40, supplemented with protease inhibitors. $4 \mathrm{C}$ libraries were prepared as described $^{69}$, with primers directed to the promoter region of Chd2 (upstream primer sequence: GCTCAAGCACCCTTTTTAAGCCAG, downstream primer sequence: AATGATACGGCGACCACCGAGATCTACACTCTTTCCCTACAC GACGCTCTTCCGATCTGAAATGTAATTTGTTCCTTTTGTC). Libraries were sequenced on Illumina NextSeq 500, and analyzed as described ${ }^{69}$.

Single-molecule FISH. Stellaris probe libraries targeting Chaserr introns (48 probes) or exons (19 probes) (Supplementary Data 3) were designed by using the Biosearch Technologies server and ordered from Biosearch Technologies. Singlemolecule FISH was done as described ${ }^{70,71}$. Briefly, mEFs were plated on poly-Llysine and $0.1 \%$ gelatin-coated coverslips. Hybridizations (25\% formamide) were done overnight with CAL Fluor ${ }^{\circledast}$ Red 590 Dye (Chaserr introns) and Quasar 670 (Chaserr exons) fluorophores. Nuclear staining was performed with DAPI (SigmaAldrich, D9542). iXon Ultra 888 EMCCD camera and Nikon's NIS-Elements were used for imaging.

Cycloheximide treatment. mESCs and mEFs were treated with DMSO (vehicle) or CHX (Sigma \#C7698) $100 \mu \mathrm{g} / \mathrm{mL}$ and collected at the indicated time points for RNA/protein analysis.

Extraction of cytoplasmic and nuclear RNA. Cells were washed twice with icecold PBS, then scraped with ice-cold buffer A (EGTA $15 \mu \mathrm{M}$, EDTA $10 \mu \mathrm{M}$, protease inhibitor cocktail (Sigma, P8340), and RNase inhibitor (ERX-E4210-01)), and centrifuged at $400 \times g, 4^{\circ} \mathrm{C}$ for $5 \mathrm{~min}$. The supernatant was discarded, fresh buffer A was added, and the pellet was mechanically pipetted with $21 \mathrm{G}$ followed by a $27 \mathrm{G}$ syringe. Cells were then centrifuged at $2000 \times g, 4^{\circ} \mathrm{C}$ for $5 \mathrm{~min}$ and the syringe step was repeated. The cells were then centrifuged at $2500 \times g, 4^{\circ} \mathrm{C}$ for $5 \mathrm{~min}$. The pellet was then kept as the nuclear fraction, and the supernatant was centrifuged again at $6000 \times g, 5$ min followed by another supernatant collection (clean cytosolic 
fraction). Nuclear pellet was then washed three times with buffer A. RNA was extracted with TRIREAGENT (MRC).

CRISPR guide RNA cloning. Guide RNAs were designed by $\mathrm{CHOPCHOP}^{72}$. Cloning of plasmids was done following Zhang Lab General Protocol (http://www. genome-engineering.org/crispr/wp-content/uploads/2014/05/CRISPR-ReagentDescription-Rev20140509.pdf) by using phU6-gRNA ${ }^{73}$ (a gift from Charles Gersbach, Addgene plasmid \#53188) or pKLV-U6gRNA(BbsI)-PGKpuro2ABFP ${ }^{74}$ (a gift from Kosuke Yusa, Addgene plasmid \#50946).

Chaserr CRISPR activation. In total, $2 \times 10^{5}$ Neuro2a cells were seeded in six-well plates and transfected with dCas9-VP64 (Addgene \#61422, at $1 \mu \mathrm{g}$ ) and five gRNAs (\#3,4,8-10, Supplementary Data 2) designed to target a region upstream of the Chaserr promoter (100 ng in total). As a control, an empty gRNA vector was used. Cells were harvested $48 \mathrm{hr}$ later and TRIZOL was used for RNA isolation.

Chaserr cloning and lentiviral production. cDNA from Source BioScience clone C130076G01 was amplified with PCR adding restriction sites for NheI at $5^{\prime}$ end and AgeI at $3^{\prime}$ end (Supplementary Data 2 ) and cloned into pLIX_402 vector (a gift from David Root, Addgene \#41394) by using restriction ligation. To produce viruses, HEK293T $\left(2.5 \times 10^{6}, 10-\mathrm{cm}\right.$ plate $)$ cells were transfected by using PEI with psPAX2 (3.5 $\mu \mathrm{g}$, a gift from Didier Trono, Addgene \#12260), pMD2.G (1.5 $\mu \mathrm{g}$, a gift from Didier Trono, Addgene, \#12259), and pLIX_402-Chaserr (5 $\mu \mathrm{g})$. Viruses were collected $48 \mathrm{hr}$ post transfection and filtered through $0.45-\mu \mathrm{m}$ sterile filters. Viruses were supplemented with polybrene (1:1000, Sigma, \#107689-10 G) upon cell infection.

3' RACE. 3' RACE was performed with RNA from WT mEFs by using SMARTer RACE 5'/3' kit (Clontech, \#634859). Briefly, RACE products were amplified by using nested primers CCCCGCTTGAAGAGTTTGAAATGGAC and GATTAC GCCAAGCTT TACCACTGAGAAATCAAGATGGCAG. Amplification resulted in a single PCR band that was then purified from $1 \%$ agarose gel by using NucleoSpin (\#740609.50), cloned into the pRACE vector (provided with the kit), and transformed into Stellar competent cells. RACE products were Sanger sequenced with M13F primer: TGTAAAACGACGGCCAGT and aligned to the mouse genome.

Micro-CT scanning and analysis. Prior to micro-CT scanning, the mice were anesthetized by using IP injection of a mix of xylazine $(10 \mathrm{mg} / \mathrm{kg})$ and ketamine $(100 \mathrm{mg} / \mathrm{kg})$. Mice were scanned by using a micro-CT device TomoScope $30 \mathrm{~S}$ Duo scanner equipped with two source-detector systems. The scanner uses two Xray sources and a detector system that are mounted on a gantry that rotates around a bed holding the animal. The operation voltages of both tubes were $40 \mathrm{kV}$. The integration time of protocols was $90 \mathrm{~ms}$ (360 rotations) for $3-\mathrm{cm}$ length, and axial images were obtained at an isotropic resolution of $80 \mu \mathrm{m}$. Due to the maximum length limit, to cover the whole mouse body, imaging was performed in two-three parts with the overlapping area, and then all slices merged to one dataset representing the entire ROI. The radiation dose range was 0.9 Gy. All micro-CT scans were reconstructed by using a filtered back-projection algorithm using scanner software. Then the reconstructed datasets for each mouse were merged to one dataset by using ImageJ software. $3 \mathrm{D}$ volume rendering images were produced by using Amira Software.

RNA immunoprecipitation (RIP). Neuro2a cells (ATCC) were collected, centrifuged at $94 \times g$ for $5 \mathrm{~min}$ at $4{ }^{\circ} \mathrm{C}$, and washed twice with ice-cold phosphatebuffered saline (PBS) supplemented with ribonuclease inhibitor $(100 \mathrm{U} / \mathrm{mL}$, \#E4210-01) and protease inhibitor cocktail (Sigma-Aldrich, \#P8340). Next, cells were lysed in $1 \mathrm{~mL}$ of lysis buffer $\left(5 \mathrm{mM}\right.$ PIPES, $200 \mathrm{mM} \mathrm{KCl}, 1 \mathrm{mM} \mathrm{CaCl}_{2}$, $1.5 \mathrm{mM} \mathrm{MgCl}_{2}, 5 \%$ sucrose, $0.5 \% \mathrm{NP}-40$, supplemented with protease inhibitor cocktail $+100 \mathrm{U} / \mathrm{ml}$ RNase inhibitor, and $1 \mathrm{mM} \mathrm{DTT}$ ) for $10 \mathrm{~min}$ on ice. Lysates were sonicated (Vibra-cell VCX-130) three times for $1 \mathrm{~s} \mathrm{ON,} 30 \mathrm{~s}$ OFF at $30 \%$ amplitude. Chilled tube holders were used and swapped between shearing runs to reduce temperature elevation. Lysates were centrifuged at $21130 \times g$ for $10 \mathrm{~min}$ at $4{ }^{\circ} \mathrm{C}$. Supernatants were then transferred to new 2-mL tubes and supplemented with $1 \mathrm{~mL}$ of IP binding/washing buffer ( $150 \mathrm{mM} \mathrm{KCl}, 25 \mathrm{mM}$ Tris (pH 7.5), $5 \mathrm{mM}$ EDTA, $0.5 \%$ NP-40, supplemented with protease inhibitor cocktail $+100 \mathrm{U} / \mathrm{ml}$ RNase inhibitor, and $0.25 \mathrm{mM}$ DTT). The samples were then rotated for $2-4 \mathrm{hr}$ at $4{ }^{\circ} \mathrm{C}$ with $5 \mu \mathrm{g}$ of antibody per reaction. Meanwhile, $50 \mu \mathrm{l}$ of beads (Dynabeads sheep anti-rat, \#11035, or GenScript A/G beads \#L00277) per reaction were washed three times with IP binding/washing buffer, followed by addition to lysates for an overnight rotating incubation. On the next day, the beads were washed three times in IP binding/washing buffer and resuspended in $0.5 \mathrm{~mL}$ of TRIZOL for RNA extraction. To generate sequencing libraries, we first depleted ribosomal RNA with Lexogen RiboCop rRNA depletion kit V1.2 (\#037), followed by Lexogen SENSE total RNA-seq library prep kit (\#042). Libraries were sequenced on NextSeq 500 by using paired-end 40-nt reads. Reads were mapped to the mouse $\mathrm{mm} 9$ genome assembly by using STAR. Exonic and intronic reads were tallied by using Picard CollectRnaSeqMetrics tool. Reads were then counted by using Homer and an annotation file constructed as described above for Pol2 ChIP-seq analysis, but containing the whole gene body of the selected isoform (from TSS to TTS). Differential expression was evaluated by using DESeq2 as implemented in Homer (as for the Pol2 ChIP-seq data).

Reporting summary. Further information on research design is available in the Nature Research Reporting Summary linked to this article.

\section{Data availability}

A reporting summary for this article is available as a Supplementary Information file. RNA-seq, ATAC-seq, ChIP-seq, RIP-seq, and 4C datasets are deposited in GEO database under the accession GSE124375. The source data underlying Figs. 1b-c, e-f, 2a-c, f-g, $3 c-j, 4 a-g, 5 a, c$, f, and 6a-f are provided as Source Data file. All data are available from the corresponding author upon reasonable request.

Received: 22 February 2019; Accepted: 18 October 2019; Published online: 08 November 2019

\section{References}

1. Iyer, M. K. et al. The landscape of long noncoding RNAs in the human transcriptome. Nat. Genet. 47, 199-208 (2015).

2. Guttman, M. et al. Chromatin signature reveals over a thousand highly conserved large non-coding RNAs in mammals. Nature 458, 223-227 (2009).

3. Kapranov, P. et al. RNA maps reveal new RNA classes and a possible function for pervasive transcription. Science 316, 1484-1488 (2007).

4. Ravasi, T. et al. Experimental validation of the regulated expression of large numbers of non-coding RNAs from the mouse genome. Genome Res. 16, 11-19 (2006).

5. Ulitsky, I. \& Bartel, DP. lincRNAs: genomics, evolution, and mechanisms. Cell 154, 26-46 (2013)

6. Perry, R. B.-T. \& Ulitsky, I. The functions of long noncoding RNAs in development and stem cells. Development 143, 3882-3894 (2016).

7. Tajul-Arifin, K. et al. Identification and analysis of chromodomain-containing proteins encoded in the mouse transcriptome. Genome Res. 13, 1416-1429 (2003).

8. Narlikar, G. J., Sundaramoorthy, R. \& Owen-Hughes, T. Mechanisms and functions of ATP-dependent chromatin-remodeling enzymes. Cell 154, 490-503 (2013)

9. Siggens, L., Cordeddu, L., Rönnerblad, M., Lennartsson, A. \& Ekwall, K. Transcription-coupled recruitment of human CHD1 and CHD2 influences chromatin accessibility and histone $\mathrm{H} 3$ and $\mathrm{H} 3.3$ occupancy at active chromatin regions. Epigenetics Chromatin 8, 4 (2015).

10. de Dieuleveult, M. et al. Genome-wide nucleosome specificity and function of chromatin remodellers in ES cells. Nature 530, 113-116 (2016).

11. Harada, A. et al. Chd2 interacts with $\mathrm{H} 3.3$ to determine myogenic cell fate. EMBO J. 31, 2994-3007 (2012)

12. Semba, Y. et al. Chd 2 regulates chromatin for proper gene expression toward differentiation in mouse embryonic stem cells. Nucleic Acids Res. 45, 8758-8772 (2017)

13. Luijsterburg, M. S. et al. PARP1 Links CHD2-Mediated Chromatin Expansion and H3.3 Deposition to DNA Repair by Non-homologous End-Joining. Mol. Cell 61, 547-562 (2016).

14. Marfella, C. G. et al. Mutation of the SNF2 family member Chd2 affects mouse development and survival. J. Cell. Physiol. 209, 162-171 (2006).

15. Dickinson, M. E. et al. High-throughput discovery of novel developmental phenotypes. Nature 537, 508-514 (2016).

16. Lamar, K.-M. J. \& Carvill, G. L. Chromatin remodeling proteins in epilepsy: lessons from CHD2-associated epilepsy. Front. Mol. Neurosci. 11, 208 (2018)

17. Shen, T., Ji, F., Yuan, Z. \& Jiao, J. CHD2 is required for embryonic neurogenesis in the developing cerebral cortex. Stem Cells 33, 1794-1806 (2015).

18. Meganathan, K. et al. Regulatory networks specifying cortical interneurons from human embryonic stem cells reveal roles for CHD2 in interneuron development. Proc. Natl Acad. Sci. USA 114, E11180-E11189 (2017).

19. Kim, Y. J. et al. Chd2 is necessary for neural circuit development and longterm memory. Neuron. 100, 1180-1193 (2019)

20. Han, P. \& Chang, C.-P. Long non-coding RNA and chromatin remodeling. RNA Biol. 12, 1094-1098 (2015).

21. Rutenberg-Schoenberg, M., Sexton, A. N. \& Simon, M. D. The properties of long noncoding RNAs that regulate chromatin. Annu. Rev. Genomics Hum. Genet. 17, 69-94 (2016).

22. Ulitsky, I., Shkumatava, A., Jan, C. H., Sive, H. \& Bartel, D. P. Conserved function of lincRNAs in vertebrate embryonic development despite rapid sequence evolution. Cell 147, 1537-1550 (2011). 
23. Lin, M. F., Jungreis, I. \& Kellis, M. PhyloCSF: a comparative genomics method to distinguish protein coding and non-coding regions. Bioinformatics 27, i275-i282 (2011)

24. Wang, L. et al. CPAT: coding-potential assessment tool using an alignmentfree logistic regression model. Nucleic Acids Res. 41, e74 (2013).

25. Kong, L. et al. CPC: assess the protein-coding potential of transcripts using sequence features and support vector machine. Nucleic Acids Res. 35, W345-W349 (2007).

26. Hezroni, H. et al. Principles of long noncoding RNA evolution derived from direct comparison of transcriptomes in 17 species. Cell Rep. https://doi.org/ 10.1016/j.celrep.2015.04.023 (2015).

27. Engreitz, J. M. et al. Local regulation of gene expression by lncRNA promoters, transcription and splicing. Nature. 539, 452-455 (2016).

28. Gilbert, L. A. et al. CRISPR-mediated modular RNA-guided regulation of transcription in eukaryotes. Cell 154, 442-451 (2013).

29. Tuck, A. C. et al. Distinctive features of lincRNA gene expression suggest widespread RNA-independent functions. Life Sci. Alliance 1, e201800124 (2018).

30. Skarnes, W. C. et al. A conditional knockout resource for the genome-wide study of mouse gene function. Nature 474, 337-342 (2011).

31. Saftig, P. et al. Impaired osteoclastic bone resorption leads to osteopetrosis in cathepsin-K-deficient mice. Proc. Natl Acad. Sci. USA 95, 13453-13458 (1998).

32. Buenrostro, J. D., Giresi, P. G., Zaba, L. C., Chang, H. Y. \& Greenleaf, W. J. Transposition of native chromatin for fast and sensitive epigenomic profiling of open chromatin, DNA-binding proteins and nucleosome position. Nat. Methods 10, 1213-1218 (2013).

33. Park, P. J. ChIP-seq: advantages and challenges of a maturing technology. Nat. Rev. Genet. 10, 669-680 (2009).

34. Skene, P. J., Hernandez, A. E., Groudine, M. \& Henikoff, S. The nucleosomal barrier to promoter escape by RNA polymerase II is overcome by the chromatin remodeler Chd1. Elife 3, e02042 (2014).

35. Perez-Perri, J. I. et al. Discovery of RNA-binding proteins and characterization of their dynamic responses by enhanced RNA interactome capture. Nat. Commun. 9, 4408 (2018).

36. Baltz, A. G. et al. The mRNA-bound proteome and its global occupancy profile on protein-coding transcripts. Mol. Cell 46, 674-690 (2012).

37. Panhale, A. et al. CAPRI enables comparison of evolutionarily conserved RNA interacting regions. Nat. Commun. 10, 2682 (2019).

38. Harada, A. et al. Generation of a rat monoclonal antibody specific for CHD2. Hybridoma 29, 173-177 (2010).

39. Lykke-Andersen, S., Ardal, B. K., Hollensen, A. K., Damgaard, C. K. \& Jensen, T. H. Box C/D snoRNP autoregulation by a cis-Acting snoRNA in the NOP56 Pre-mRNA. Mol. Cell 72, 99-111.e5 (2018).

40. Jangi, M., Boutz, P. L., Paul, P. \& Sharp, P. A. Rbfox2 controls autoregulation in RNA-binding protein networks. Genes Dev. 28, 637-651 (2014).

41. Triboulet, R. \& Gregory, R. I. Autoregulatory Mechanisms Controlling the Microprocessor. in Regulation of microRNAs (ed. Großhans, H.) 56-66 (Springer, US, 2010).

42. Luo, W. et al. The conserved intronic cleavage and polyadenylation site of CstF-77 gene imparts control of 3' end processing activity through feedback autoregulation and by U1 snRNP. PLoS Genet. 9, e1003613 (2013).

43. $\mathrm{Li}, \mathrm{Y}$. et al. An intron with a constitutive transport element is retained in a Tap messenger RNA. Nature 443, 234-237 (2006).

44. Pendleton, K. E. et al. The U6 snRNA m6A methyltransferase METTL16 regulates SAM synthetase intron retention. Cell 169, 824-835.e14 (2017).

45. Feng, Y., Sansam, C. L., Singh, M. \& Emeson, R. B. Altered RNA editing in mice lacking ADAR2 autoregulation. Mol. Cell. Biol. 26, 480-488 (2006).

46. Crews, S. T. \& Pearson, J. C. Transcriptional autoregulation in development. Curr. Biol. 19, R241-R246 (2009).

47. Pruneski, J. A., Hainer, S. J., Petrov, K. O. \& Martens, J. A. The Paf1 complex represses SER3 transcription in Saccharomyces cerevisiae by facilitating intergenic transcription-dependent nucleosome occupancy of the SER3 promoter. Eukaryot. Cell 10, 1283-1294 (2011).

48. Martens, J. A., Laprade, L. \& Winston, F. Intergenic transcription is required to repress the Saccharomyces cerevisiae SER3 gene. Nature 429, 571-574 (2004).

49. Martens, J. A., Wu, P.-Y. J. \& Winston, F. Regulation of an intergenic transcript controls adjacent gene transcription in Saccharomyces cerevisiae. Genes Dev. 19, 2695-2704 (2005).

50. Hainer, S. J., Pruneski, J. A., Mitchell, R. D., Monteverde, R. M. \& Martens, J. A. Intergenic transcription causes repression by directing nucleosome assembly. Genes Dev. 25, 29-40 (2011).

51. Thebault, P. et al. Transcription regulation by the noncoding RNA SRG1 requires Spt2-dependent chromatin deposition in the wake of RNA polymerase II. Mol. Cell. Biol. 31, 1288-1300 (2011).

52. Cho, S. W. et al. Promoter of lncRNA gene PVT1 is a tumor-suppressor DNA boundary element. Cell 173, 1398-1412.e22 (2018).
53. Nagarajan, P. et al. Role of chromodomain helicase DNA-binding protein 2 in DNA damage response signaling and tumorigenesis. Oncogene 28, 1053-1062 (2009).

54. Kulkarni, S. et al. Disruption of chromodomain helicase DNA binding protein 2 (CHD2) causes scoliosis. Am. J. Med. Genet. A 146A, 1117-1127 (2008).

55. Feyder, M. \& Goff, L. A. Investigating long noncoding RNAs using animal models. J. Clin. Invest. 126, 2783-2791 (2016).

56. Ulitsky, I. Evolution to the rescue: using comparative genomics to understand long non-coding RNAs. Nat. Rev. Genet. https://doi.org/10.1038/nrg.2016.85 (2016).

57. Jozefczuk, J., Drews, K. \& Adjaye, J. Preparation of mouse embryonic fibroblast cells suitable for culturing human embryonic and induced pluripotent stem cells. J. Vis. Exp. https://doi.org/10.3791/3854 (2012).

58. Ying, Q.-L., Stavridis, M., Griffiths, D., Li, M. \& Smith, A. Conversion of embryonic stem cells into neuroectodermal precursors in adherent monoculture. Nat. Biotechnol. 21, 183-186 (2003).

59. Durocher, Y., Perret, S. \& Kamen, A. High-level and high-throughput recombinant protein production by transient transfection of suspensiongrowing human 293-EBNA1 cells. Nucleic Acids Res. 30, E9 (2002).

60. Dobin, A. et al. STAR: ultrafast universal RNA-seq aligner. Bioinformatics 29, 15-21 (2013)

61. Li, B. \& Dewey, C. N. RSEM: accurate transcript quantification from RNASeq data with or without a reference genome. BMC Bioinforma. 12, 323 (2011).

62. Love, M., Anders, S. \& Huber, W. Differential analysis of count data-the DESeq2 package. Genome Biol. 15, 550 (2014).

63. Blecher-Gonen, R. et al. High-throughput chromatin immunoprecipitation for genome-wide mapping of in vivo protein-DNA interactions and epigenomic states. Nat. Protoc. 8, 539-554 (2013).

64. Langmead, B., Trapnell, C., Pop, M. \& Salzberg, S. L. Ultrafast and memoryefficient alignment of short DNA sequences to the human genome. Genome Biol. 10, R25 (2009).

65. Zhang, Y. et al. Model-based analysis of ChIP-Seq (MACS). Genome Biol. 9, R137 (2008).

66. Quinlan, A. R. \& Hall, I. M. BEDTools: a flexible suite of utilities for comparing genomic features. Bioinformatics 26, 841-842 (2010).

67. Heinz, S. et al. Simple combinations of lineage-determining transcription factors prime cis-regulatory elements required for macrophage and B cell identities. Mol. Cell 38, 576-589 (2010).

68. Olivares-Chauvet, $\mathrm{P}$. et al. Capturing pairwise and multi-way chromosomal conformations using chromosomal walks. Nature 540, 296-300 (2016).

69. Schwartzman, O. et al. UMI-4C for quantitative and targeted chromosomal contact profiling. Nat. Methods 13, 685-691 (2016).

70. Itzkovitz, S. et al. Single-molecule transcript counting of stem-cell markers in the mouse intestine. Nat. Cell Biol. 14, 106-114 (2011).

71. Raj, A., van den Bogaard, P., Rifkin, S. A., van Oudenaarden, A. \& Tyagi, S. Imaging individual mRNA molecules using multiple singly labeled probes. Nat. Methods 5, 877-879 (2008).

72. Montague, T. G., Cruz, J. M., Gagnon, J. A., Church, G. M. \& Valen, E. CHOPCHOP: a CRISPR/Cas9 and TALEN web tool for genome editing. Nucleic Acids Res. 42, W401-W407 (2014).

73. Kabadi, A. M., Ousterout, D. G., Hilton, I. B. \& Gersbach, C. A. Multiplex CRISPR/Cas9-based genome engineering from a single lentiviral vector. Nucleic Acids Res. 42, e147 (2014).

74. Koike-Yusa, H., Li, Y., Tan, E. P., Velasco-Herrera Mdel, C. \& Yusa, K. Genome-wide recessive genetic screening in mammalian cells with a lentiviral CRISPR-guide RNA library. Nat. Biotechnol. 32, 267-273 (2014).

75. Consortium, Fantom et al. A promoter-level mammalian expression atlas. Nature 507, 462-470 (2014)

76. Nam, J. W. et al. Global analyses of the effect of different cellular contexts on microRNA targeting. Mol. Cell 53, 1031-1043 (2014).

77. Fagerberg, L. et al. Analysis of the human tissue-specific expression by genome-wide integration of transcriptomics and antibody-based proteomics. Mol. Cell. Proteom. 13, 397-406 (2014).

78. Li, B. et al. A comprehensive mouse transcriptomic BodyMap across 17 tissues by RNA-seq. Sci. Rep. 7, 4200 (2017).

79. de Wit, E., Braunschweig, U., Greil, F., Bussemaker, H. J. \& van Steensel, B. Global chromatin domain organization of the Drosophila genome. PLoS Genet. 4, e1000045 (2008)

\section{Acknowledgements}

We thank Noam Stern-Ginossar, Schraga Schwarz, Shalev Itzkovitz, Alena Shkumatava, Yaqub Hanna, Eran Meshorer, and Ulitsky lab members for helpful discussions and comments on the paper. Damari Golda, Haffner-Krausz Rebecca, and Maizenberg Alina for mouse transgenics. Ori Brenner for assistance with histopathology. Dana Hirsh and 
Raya Eilam for assistance with single-molecule FISH. This research was supported by the Israeli Centers for Research Excellence (1796/12); Israel Science Foundation (1242/14 and 1984/14); European Research Council project lincSAFARI; Nella and Leon Benoziyo Center for Neurological Disease; The Helen and Martin Kimmel Institute for Stem Cell Research; Lapon Raymond; the Abramson Family Center for Young Scientists. I.U. is incumbent of the Sygnet Career Development Chair for Bioinformatics.

\section{Author contributions}

A.R. and I.U. conceived the study. A.R. performed most experiments and analyzed the data together with I.U. L.M., N.G., M.J.G., R.B.T.P., R.K., M.G. and I.B. performed additional experiments and assisted with interpretation of the results. A.R. and I.U. wrote the paper with input from all other authors.

\section{Competing interests}

The authors declare no competing interests.

\section{Additional information}

Supplementary information is available for this paper at https://doi.org/10.1038/s41467019-13075-8.

Correspondence and requests for materials should be addressed to I.U.
Peer review information Nature Communications thanks the anonymous reviewers for their contribution to the peer review of this work. Peer reviewer reports are available.

Reprints and permission information is available at http://www.nature.com/reprints

Publisher's note Springer Nature remains neutral with regard to jurisdictional claims in published maps and institutional affiliations.

(c) (i)

Open Access This article is licensed under a Creative Common Attribution 4.0 International License, which permits use, sharing adaptation, distribution and reproduction in any medium or format, as long as you give appropriate credit to the original author(s) and the source, provide a link to the Creative Commons license, and indicate if changes were made. The images or other third party material in this article are included in the article's Creative Commons license, unless indicated otherwise in a credit line to the material. If material is not included in the article's Creative Commons license and your intended use is not permitted by statutory regulation or exceeds the permitted use, you will need to obtain permission directly from the copyright holder. To view a copy of this license, visit http://creativecommons.org/ licenses/by/4.0/.

(c) The Author(s) 2019 